\title{
A Deformation-Sensing Augmented Stylus
}

\author{
by
}

Nicholas Fellion

A thesis submitted to the Faculty of Graduate and Postdoctoral Affairs in partial fulfillment of the requirements for the degree of

Masters of Arts

in

Human Computer Interaction

Carleton University

Ottawa, Ontario

(C) 2016, Nicholas Fellion 


\begin{abstract}
Digital artists frequently use augmented styluses to modify parametric input such as stroke width. We contribute to this domain by creating a flexible stylus prototype, the FlexStylus, that detects deformation as a force vector with both an angle and an absolute value, providing two degrees of freedom for improving the experience of digital artists.

We outline the construction of the prototype and the principles behind the sensing method, which uses a cluster of four fibre-optic flexion sensors. We propose interaction techniques using the FlexStylus to improve menu navigation and tool selection. Finally, we describe a study measuring accuracy during dynamic parameter adjustment. We compare users' ability to match a changing target value using a commercial pressure stylus and the FlexStylus' absolute deformation. When using the FlexStylus, users had a significantly higher accuracy overall. This suggests that deformation may be a useful input method for future work considering stylus augmentation.
\end{abstract}




\section{Acknowledgements}

My supervisor, Audrey Girouard, showed great patience and offered excellent guidance at every stage of the project. I cannot stress enough how much credit goes to her, and how lucky I am to have worked with her. Her excellent eye for clarity of communication, not to mention ideas, has been essential throughout the project.

Thomas Pietrzak, my adoptive supervisor, along with rest of MJOLNIR at Université Lille 1/INRIA, taught me a great deal. It was too brief, but I will remember my time there as very formative. There is not enough space to mention everyone, but special thanks goes to Sylvain Malacria for never letting my ideas off easy, Izzatbek Mukhanov for encouraging me to challenge myself, and Alix Goguey for being an exceptional fellow. My gratitude goes to MITACS and Campus-France, whose joint funding enabled the above collaboration, as well the Hendrika Alice Eisen Memorial Foundation for providing funding for me to present my work at CHI 2016. I thank Alexander Keith Eady at Carleton for his support throughout, from creating a software application for the first iteration of the prototype, to providing moral support and muchneeded statistics advice towards the end. Our sincerest gratitude also goes to Sonia Chiasson for lending a tablet at a moment of need. Had I not met Roel Vertegaal, I would not be where I am. I cannot thank him enough for introducing me to HCI.

I would also like to thank my friends for their encouraging words, their help, and their willingness to listen. Most of all, thanks go to my family: my brother, Matthew Fellion, and my parents, David and Catherine Fellion. They were with me through the most stressful times, and their unflagging support saw me through. 


\section{Table of Contents}

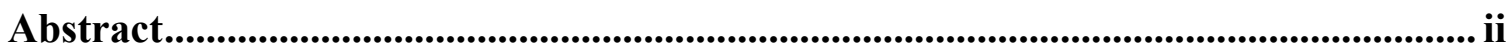

Acknowledgements .......................................................................................................................iii

Table of Contents .......................................................................................................... iv

List of Tables .................................................................................................................. viii

List of Illustrations...................................................................................................... ix

List of Appendices................................................................................................................. xi

1 Chapter: Introduction ........................................................................................................ 1

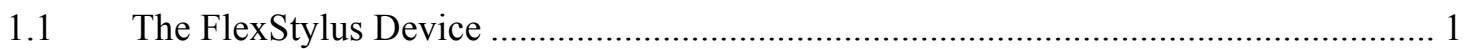

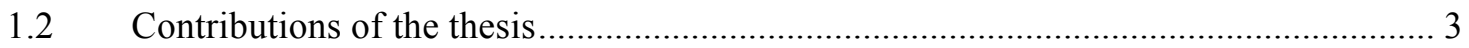

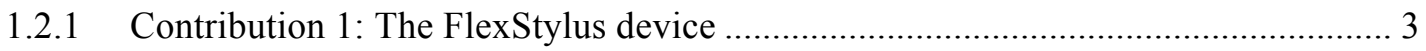

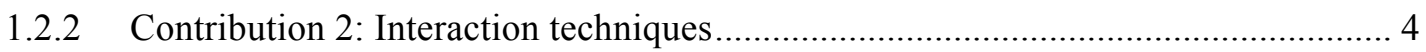

1.2.3 Contribution 3: Comparative Study Between Flex and Pressure Styluses ............... 4

2 Chapter: Related Work ...........................................................................................

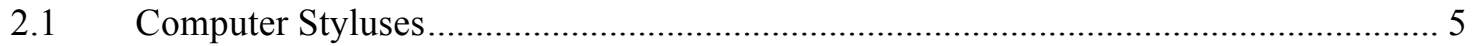

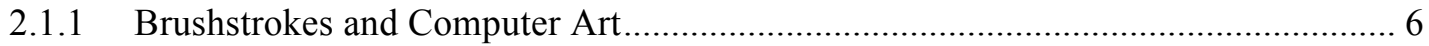

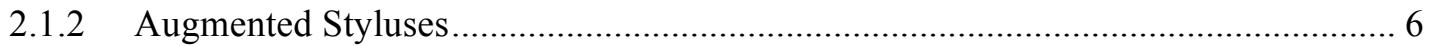

2.1.3 Augmentation for Parametric Control of brushstrokes .......................................... 7

2.1.4 Augmentation for Pointing and Menu Navigation............................................... 7

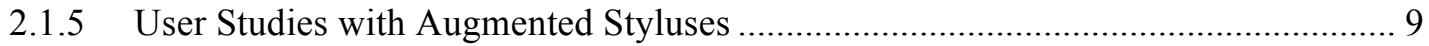

2.1.6 Context/grip sensitivity using a stylus device .................................................. 10

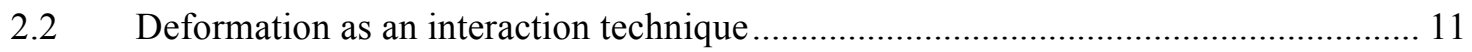

2.2.1 Non-Planar Deformation using fibre-optic flex sensing ....................................... 13

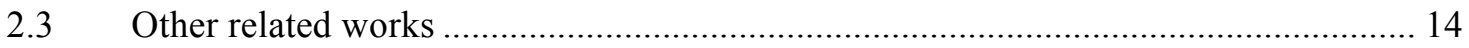




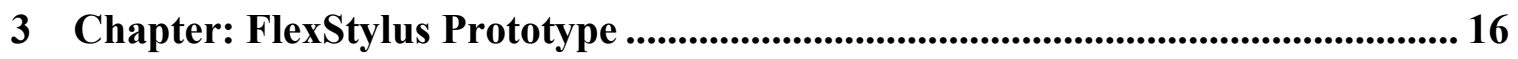

3.1 Limitations of currently used flex sensing technologies ......................................... 16

3.2 Outline of flex sensing technique for flexible stylus.......................................... 17

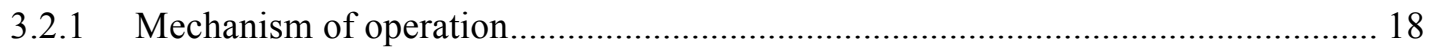

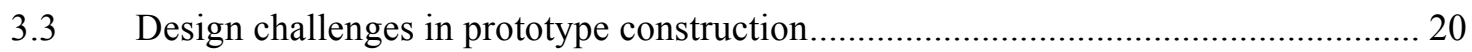

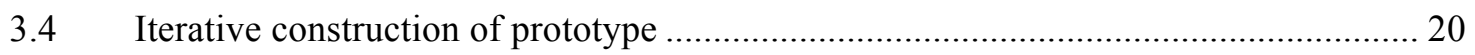

3.4.1 Non-functional mockups, interaction sketching, and sensor proof of concept........ 20

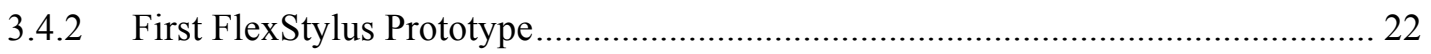

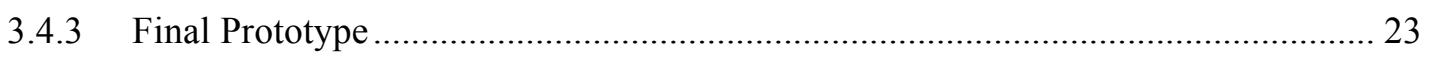

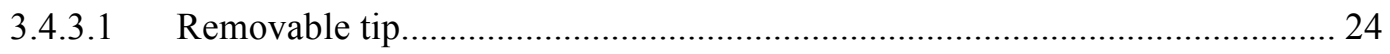

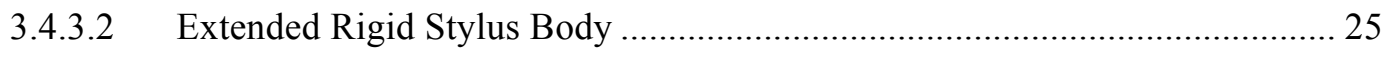

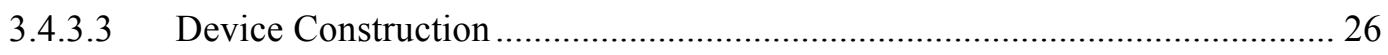

4 Chapter: Interaction Techniques .................................................................... 28

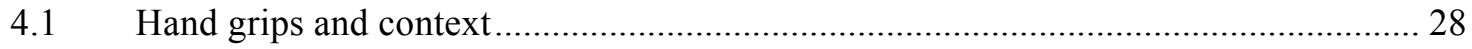

4.2 The Tool Grip: Drawing and Context Menus........................................................... 29

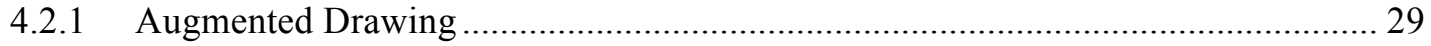

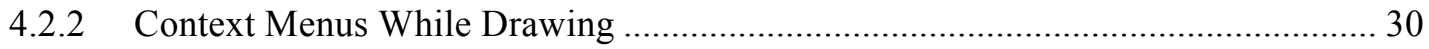

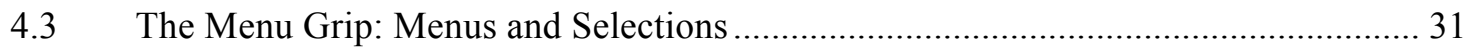

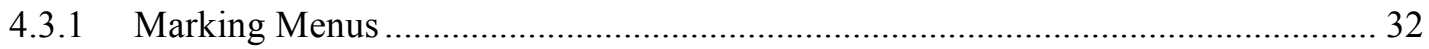

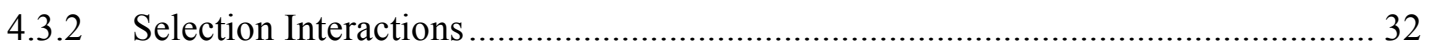

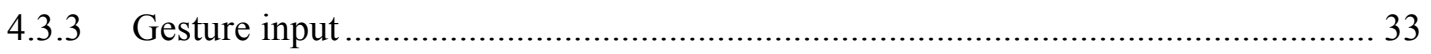

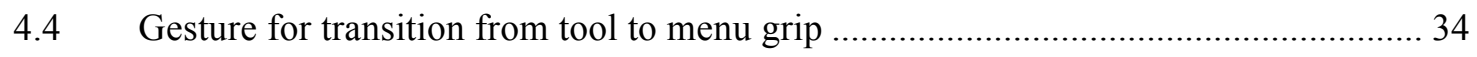

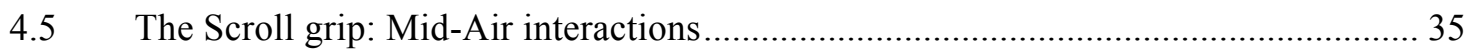

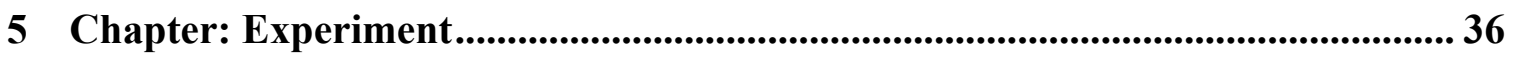

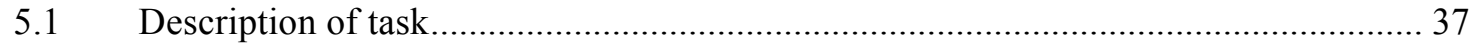

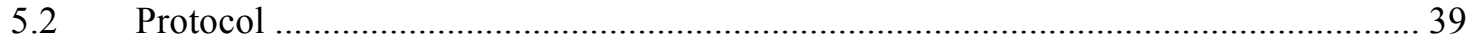




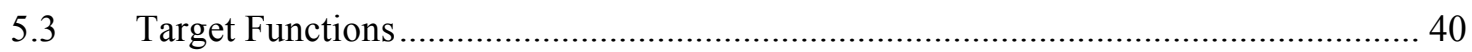

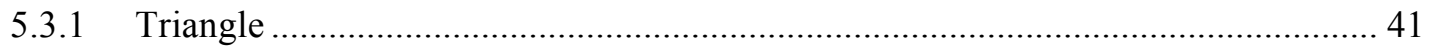

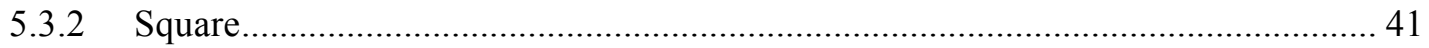

5.3.3 Sine

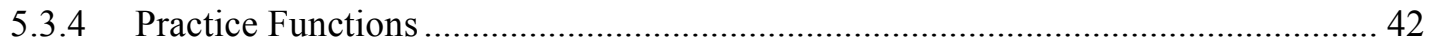

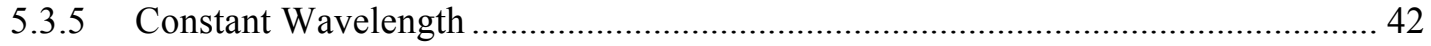

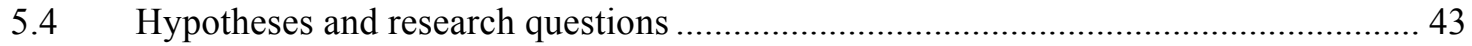

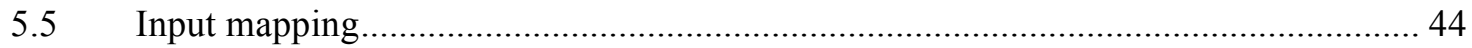

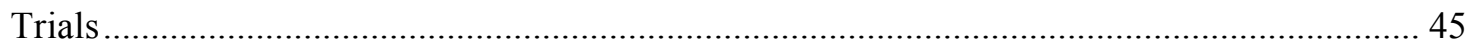

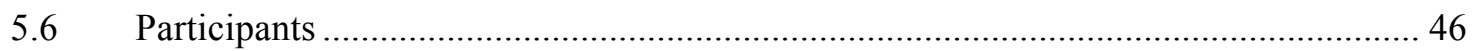

6 Chapter: Results............................................................................................................... 47

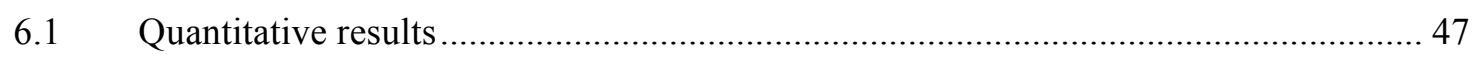

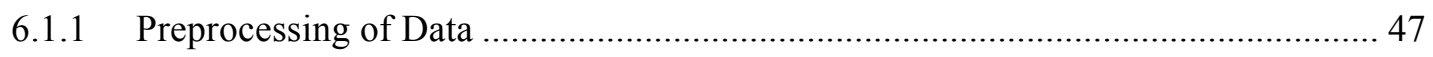

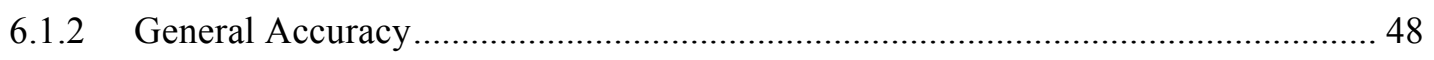

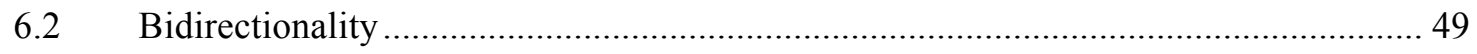

6.3 Square wave condition: static and dynamic accuracy .......................................... 50

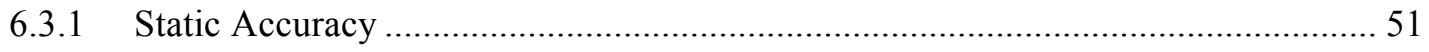

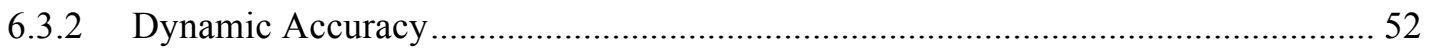

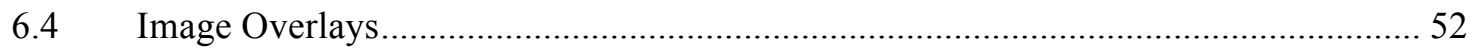

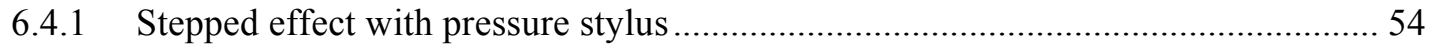

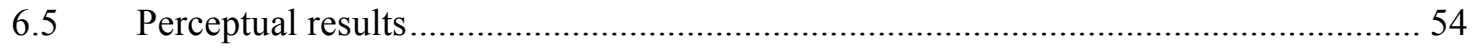

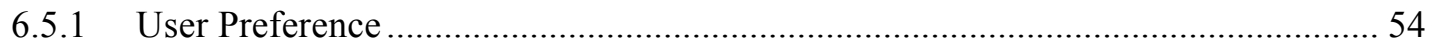

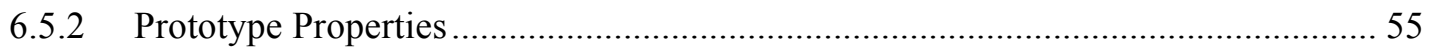

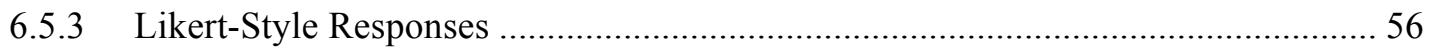

7 Chapter: Discussion ................................................................................................... 58

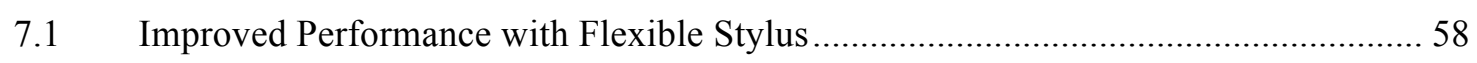




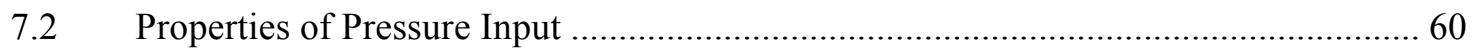

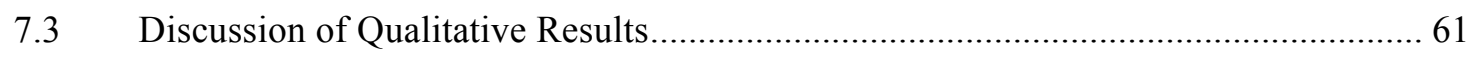

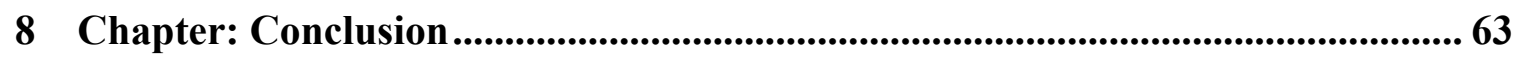

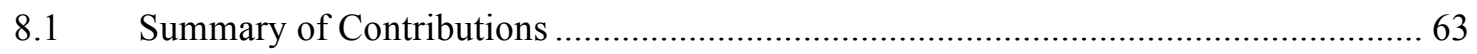

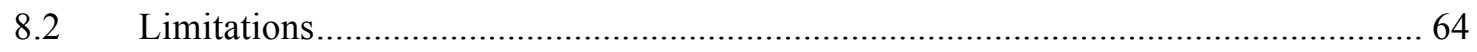

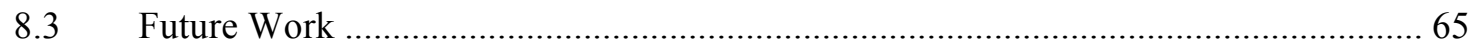

Appendices .............................................................................................................................. 68

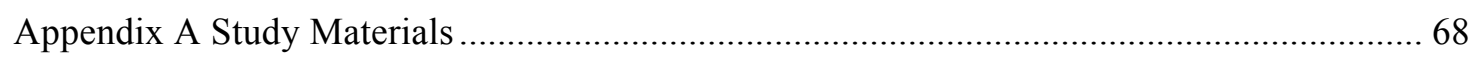

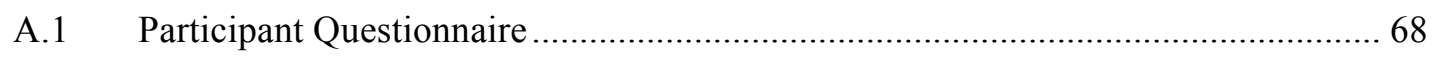

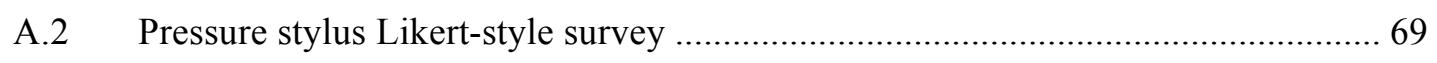

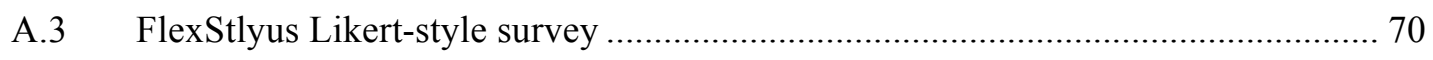

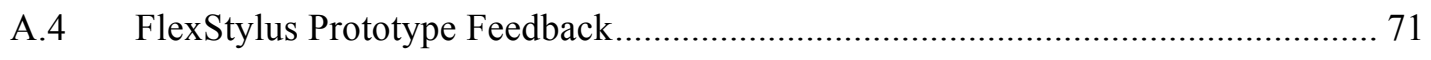

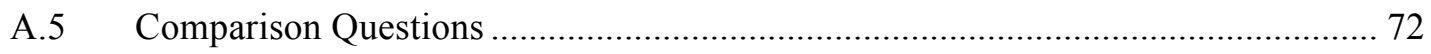

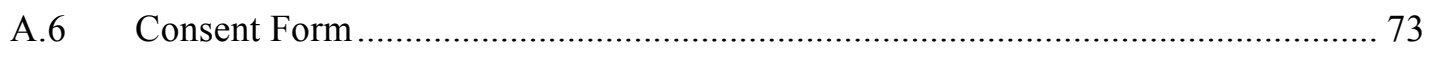

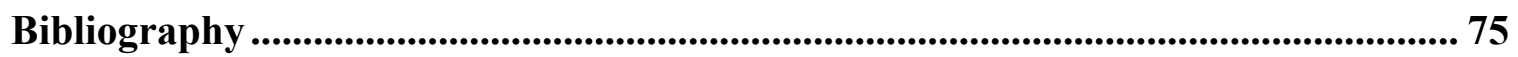




\section{List of Tables}

Table 1: Counts and percentages of participant device preference................................. 55

Table 2: Participant Responses regarding physical properties of FlexStylus prototype... 56 


\section{List of Illustrations}

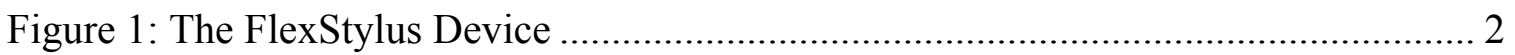

Figure 2: The ShapeTape System [4]................................................................... 14

Figure 3: Herot and Weinzapfel's pressure sensitive display [21], with force vector ...... 15

Figure 4: FlexPoint resistive bend sensor ....................................................................... 16

Figure 5: Fibre-optic Sensor from Twend prototype, showing abrasion ........................... 17

Figure 6: Degrees of deformation freedom detected by FlexStylus ............................... 18

Figure 7: Mechanism of operation of flex sensor cluster ............................................... 19

Figure 8: Relative voltage outputs of bend sensors during circle gesture, showing PCB

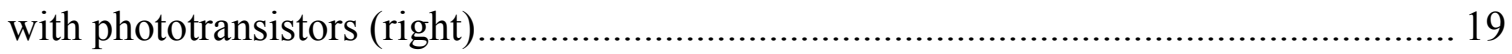

Figure 9: Initial sketches (left) and clay mockup prototype (right) ................................. 21

Figure 10: Cutaway of First Flexstylus prototype ……………….................................. 22

Figure 11: Photo of First Flexstylus prototype …………………………………....... 23

Figure 12: Cutaway of Removable Tip...................................................................... 25

Figure 13: Final prototype body design, showing phototransistor circuit ........................ 26

Figure 14: (left to right) Tool Grip, Menu Grip, Scroll Grip ........................................... 28

Figure 15: Using deformation to control stroke width................................................ 30

Figure 16: Selecting Drawing tools with Radial Menu ................................................ 32

Figure 17: Selecting from a continuous spectrum with the menu grip ............................. 33

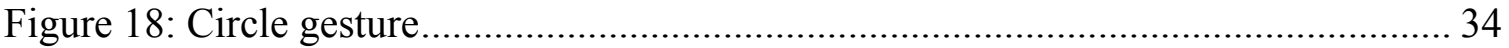

Figure 19: Study Apparatus, pressure stylus trial, triangular curve................................. 37 
Figure 20: Study setup (left), study detail (right) Arrow indicates function scrolling direction

Figure 21:Test functions: triangle (top), square (middle) and sine (bottom), showing small (left), medium (middle) and large (right) sizes. The pixel values indicated are calculated from the center of the function.

Figure 22: Boxplots of distance from target collapsed across factors, by participant ...... 48 Figure 23: Boxplots of distance from target by input device and function, collapsed across size

Figure 24: Estimated Marginal Means of distance from target by slope (sine and triangle

functions) 50

Figure 25: Square function, showing areas studied for static (blue) and dynamic (red) accuracy

Figure 26: Superimposed trial images, pressure stylus (left) and FlexStylus (right), medium size condition, for the triangle (top), square (middle), and sine (bottom) functions.

Figure 27: Trial data for one typical participant demonstrating stepped pattern with the pressure stylus (left). The same participant is not demonstrating the pattern when using

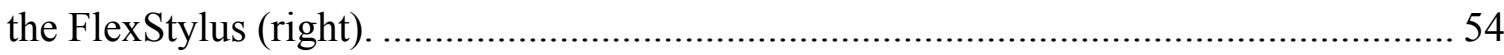

Figure 28: Graph of Likert Responses ................................................................ 56 


\section{List of Appendices}

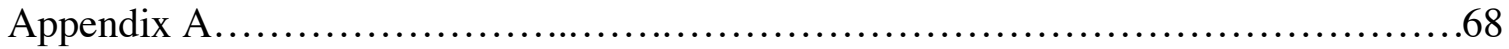

A.1 Participant Questionnaire.........................................69

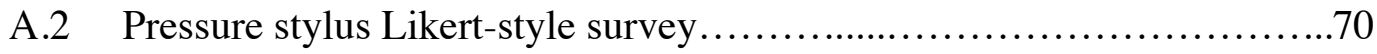

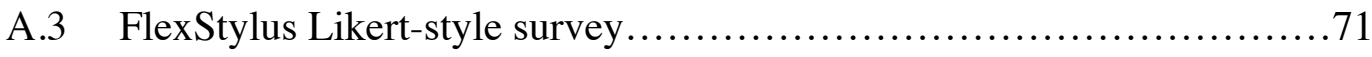

A.4 FlexStylus Prototype feedback................................. 72

A.5 Comparison Questions...........................................73

A.6 Consent Form.................................................. 74 


\section{Chapter: Introduction}

It is easy to imagine a clear divide between non-digital and digital artistic media. On one side of this divide, there is the "physical media"-everything from charcoal to fresco, gouache to oils. On the other side is "digital media" - applications for 3D modelling, vector illustration, simulated painting, or photo manipulation. People often associate certain affective qualities to the different sides, using various intangible descriptors: One might perceive that "real painting" has warmth, contrasted with the "coldness" of digital art, that or non-digital art evokes an immediacy that is inaccessible to the more mediated world of digital art.

However, it is important to remember that human creative production is closely connected to the constantly evolving spectrum of technologies available to us. Consider the camera obscura, first described as a drawing aid in 1558 , which enabled painting scenes more accurately by projecting the subject matter onto a wall using a pinhole [9].

There has been a constant progression of artistic technology throughout history, with varying goals, which might include enabling works to be created that were previously impossible, creating works more quickly, and myriad possible novel creative effects. Our work is part of this continuum of ever-developing artistic technology. Rather than seeing a gap between the digital and physical media, we are interested in how technology can allow an artist make use of, in a digital context, the subtle physical manipulation of the hand and artistic tool generally associated with traditional media.

\subsection{The FlexStylus Device}

As suggested by the name, the FlexStylus is a flexible stylus (Figure 1). It is shaped like a standard computer stylus - i.e., it is shaped like a pen or brush-but uses 
flexion detecting sensors to determine the angle, as well as the degree of flexion applied to the device. The user might deform the device in a variety of ways, as by posing the tip against the hard surface of the tablet and applying force to the side of the device, or by holding the device like a pen and manipulating it with the thumb. The Flexstylus detects deformation input as a vector-it measures the absolute amplitude of the deformation, as well as the angle of deformation relative to the body of the pen.

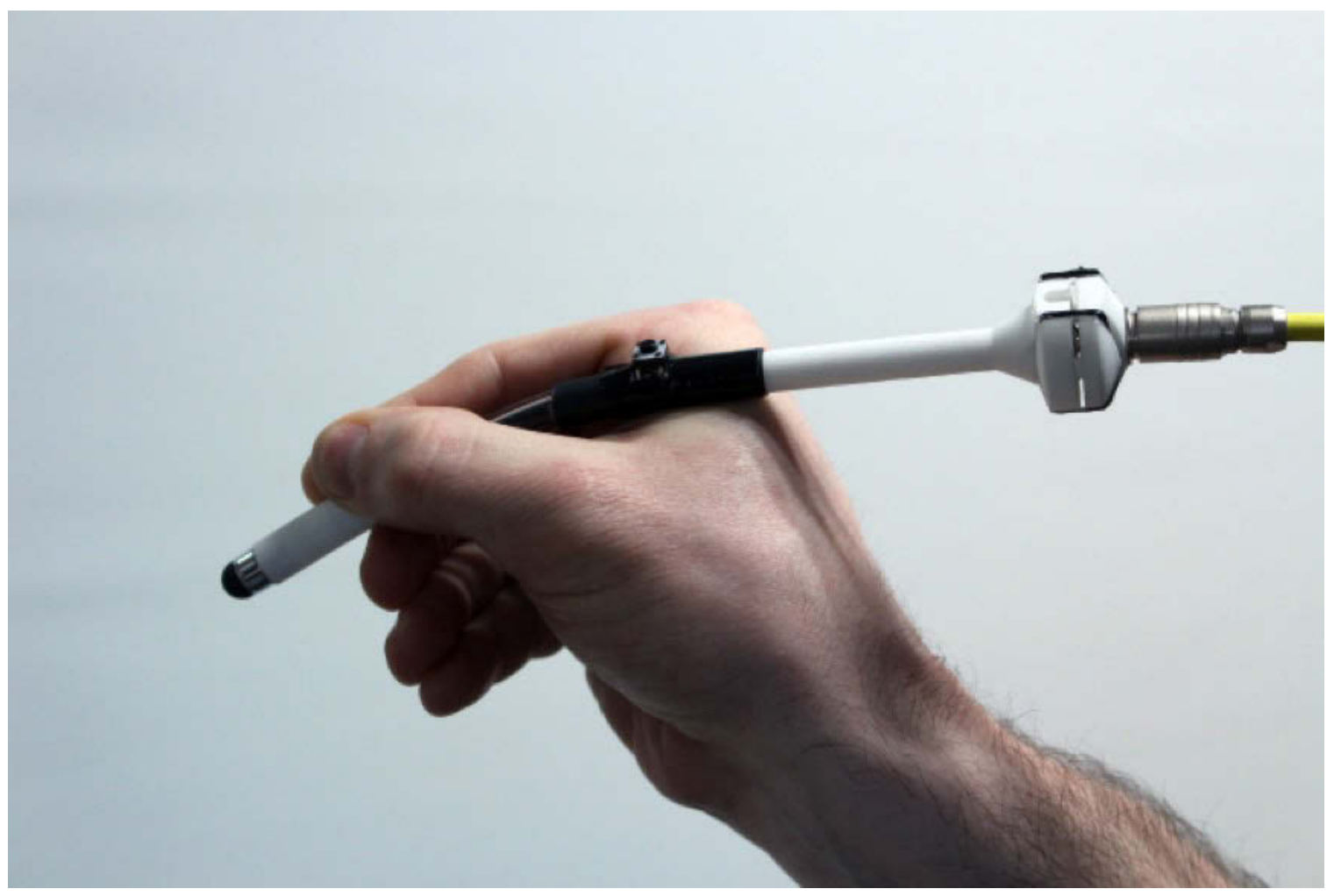

Figure 1: The FlexStylus Device

The FlexStylus is not designed to replicate a physical artistic tool, although this can be a useful metaphor for explaining the device. Instead, the FlexStylus is an input device designed to take advantage of types of hand movements previously unexplored in the domain of stylus-type devices. The way that these hand movements, tensions, extensions, and displacements can be used are defined here for example purposes, and for the purposes of evaluating the device. The FlexStylus is not limited to these uses, and 
indeed, as an artistic device, it would be ineffective if any limitations were imposed on the way it might be used. For example, the deformation sensing of the FlexStylus allows the user to imitate a brush, but the device is not designed to only simulate the use of a brush. It is designed to be its own tool, that transfers some of the physicality of traditional art media (e.g. the feeling of resistance provided by brush bristles on a page), to a digital context. The artist can make use of resulting extra input capabilities, as well as the resulting haptic feedback, as they wish.

\subsection{Contributions of the thesis}

This thesis offers 3 contributions: the development of the device itself, the interaction techniques associated with it, and the results of a study conducted on user flexion input performance with the FlexStylus.

\subsubsection{Contribution 1: The FlexStylus device}

Chapter 3 explains the construction of the device, which employs a novel kind of flexion sensing which, to date, has not been widely considered in prior research on flexible input devices. Research on flexible devices has largely been limited to devices with slate form-factors, which we contend to be due to the limited kinds of flexion sensing tools available to researchers. We describe the technique for flexion sensing, employing a cluster of 4 fibre-optic flexion sensors to derive a measurement of absolute and angular flexion from the device. We also describe the steps we took in designing and building the prototype, which we created using rapid-prototyping techniques and processes. The technology that enables the FlexStylus form factor and its two degrees of input freedom can be applied to the development of other devices, expanding the domain of flexible device research into new form factors and input methods. 


\subsubsection{Contribution 2: Interaction techniques}

Chapter 4 outlines eight interaction techniques that make use of the FlexStylus' vector deformation input. Because there are large differences between users' input freedoms depending on grip, we categorize these interaction techniques into 3 grips: the tool grip, the menu grip, and the scroll grip. This list of interaction techniques is not exhaustive. However, it describes certain ways that incorporating flexion into a stylus might help computer artists be more effective and expressive in their disciplines. It also outlines uses for the FlexStylus beyond the artistic domain - how it may extend stylusbased menu and navigation interactions more broadly.

\subsubsection{Contribution 3: Comparative Study Between Flex and Pressure Styluses}

Chapter 5 presents a first evaluation of the FlexStylus, which focuses on users' ability to match a target input amplitude, comparing pressure and flexion as parametric input methods. We explain the study design, construction, and execution. Chapter 6 presents a summary of the results of the study. Chapter 7 discusses the ramifications and meanings of the study results for the flexible stylus prototype, and how these principles might be able to be applied in future, outside of the context of parametric stroke modification, and to augmented styluses in general. 


\section{Chapter: Related Work}

Research involving the FlexStylus builds predominantly on two areas of prior work: augmented styluses and deformable user interfaces. This section begins with a brief overview of the general place of the computer stylus within computing history, and narrows towards the two aspects of computer styluses which have most relevance to the FlexStylus system: styluses that are augmented with additional degrees of freedom to aid in parametric control of artistic brushes, and augmented styluses used for menu navigation. Following, this section will discuss the history of systems incorporating flexion as an input method. Finally, it will discuss other related works that have informed our research, but do not fall neatly into the above categories.

\subsection{Computer Styluses}

The first example of electric encoding of pen input is the Telautograph, patented in 1888 [18]. This device transmitted pen input over telegraph lines. Robert Everett is acknowledged to have developed the precursor to the modern stylus with the light gun in the 1950s [32]. This light gun was later used in the SAGE Air Defense Information system as a graphical selection device[32]. The most famous early stylus implementation, and the most relevant, as it deals with the stylus as a device for generating computer graphics, is Ivan Sutherland's Sketch Pad [44]. Sketch Pad was a light-pen CAD system, which focused on ways in which the computer system could augment the user's capacities using geometric algorithms. However, in the Sketch Pad paper, use of the penbased system for artistic purposes receives scant mention, with the authors instead focusing on architectural planning, circuit design, and other CAD-related applications. Sketch Pad, however, was the first system to articulate and demonstrate the benefits of 
computer aided drawing, including being able to save and non-destructively permute saved drawings.

\subsubsection{Brushstrokes and Computer Art}

In 1986, Strassmann described a system for rendering brushstrokes in computer art [41]. While this system used a mouse and a keyboard as input devices for the coordinates and pressure of the brushstroke respectively, Strassmann stressed that the prototype system would be improved by using a different input device, proposing a stylus or touchpad to increase the similarity to painting. While not implemented with a stylus, this system, using simultaneous position input with a mouse and stroke pressure with a keyboard, did contain a theme that would continue in computer-art related research: the ability to simultaneously control the brush coordinates and the brushstroke properties.

While researchers have done substantial work on realistic brushstroke rendering, these projects are outside the scope of our research-we are interested in the ability to accurately input parametric information, which is not related to any particular strokerendering software system.

\subsubsection{Augmented Styluses}

In order to improve the experience of stylus users, researchers and device manufacturers have created devices that augment styluses with one or more extra degrees of freedom. Previous research on augmented styluses focused on solving two problems. The first problem is that with a standard $\mathrm{x}-\mathrm{y}$ stylus, users are unable to simultaneously manipulate parameters other than the path of the stylus tip. This is a considerable disadvantage compared to physical art media, where an artist is able to manipulate a wide variety of parameters simultaneously to the path of the drawing tool, such as the angle of 
the pen nib, the tilt of a brush, or the amount of pressure applied. The second goal of augmenting stylus' $x-y$ input is to expand the degrees of freedom available for users in menu, selection, and navigation contexts, in order to enhance user control and solve various problems associated with stylus input. While styluses and applications that provide an extra degree of freedom for altering stroke parameters are popular commercial devices, systems using stylus augmentation for menu and selection tasks, despite initial research, are still relatively rare in widely adopted commercial applications.

\subsubsection{Augmentation for Parametric Control of brushstrokes}

The stroke qualities of a basic, non-augmented, $x-y$ stylus cannot be varied dynamically while the stroke is in progress. While discussing touch pads, Kurtenbach et al. suggested solving this problem by continuously varying pressure levels in order to map the width of a brushstroke [28]. Stylus augmentations such as pressure sensing or tilt sensing also attempt to solve this problem by providing loose physical analogues to the behaviour of traditional media. Pressure is the most common and commercially developed form of stylus augmentation, and can be found in devices such as Wacom styluses [51] and the Apple Pencil [2], the latter also detecting tilt. These devices tend to use the additional degrees of freedom for the purpose of parametric control rather than navigation, e.g. pressure for nib size and tilt to represent the tilt of a pastel crayon. This work has graduated from the research stage, and is found in a wide variety of commercial devices.

\subsubsection{Augmentation for Pointing and Menu Navigation}

Researchers have turned their attention to using the augmentation of the stylus as another degree of freedom for aiding in menu interaction. Ramos et al. [33] proposed 
numerous techniques for improving menu navigation with pressure, specifically augmenting stylus gestures with pressure to create "pressure marks". They were interested in providing a solution to the modal problem described by Saund and Lank [37], which stems from the fact that the stylus serves multiple input functions; it is simultaneously a pointer for drawing, as well as other non-drawing tasks, such as performing selections. Ramos et al. [35] also proposed numerous methods of laying out menu items for pressure input, and created a novel method of combining pressure and $x-y$ input for scrolling tasks, called Zliding [34]. With the Zlider widget, a user employs $\mathrm{x}-\mathrm{y}$ input on a scroll bar for scrolling, while at the same time manipulating the granularity of effect of that $x-y$ input using stylus pressure.

In a research whitepaper, Huot et al. [24] created a menu navigation tool that uses stylus pressure to further differentiate a series of pie menus. Their goal was to increase the user's level of control over a pie menu, and thus increase the number of potential options associated with that menu. They found they could use 2 levels of pressure to increase a marking menu to 16 items from 8 .

Xin et al. [56] suggested that augmenting styluses with tilt can improve eyes-free interaction, because a user can be aware of how they are tilting a stylus without looking. Bi et al. [5] implemented roll detection on a stylus to enable roll-based interactions such as menu item selection and scrolling. Tian et al. [47] created a prototype system which made use of tilt to allow the user to navigate through a pie menu using the tilt of the stylus. 
The proliferation of papers of this kind points to a common occupation with a particular question: How can the fine control over the hand associated with stylus and pen usage be further exploited for navigation and menu interactions?

\subsubsection{User Studies with Augmented Styluses}

To accompany research on the applications of augmented styluses, researchers have performed a number of studies in order to determine exactly the strengths and weaknesses of pressure and tilt as input mechanisms.

In order to better develop pressure-based menus, Ramos et al. [35] conducted a study to determine a number of properties of pressure-based styluses. They asked users to perform pressure gestures, where the amount of pressure required to perform the gesture successfully was represented as a target. They found that the error rate and the number of crossings (how many times a user had to cross out of and back into the boundaries of a target) both increased above 6 discrete levels of pressure input. Their work suggests that the threshold of differentiability, below which it is difficult for users to distinguish between levels of pressure, is relatively large for pressure as input. This threshold is a larger concern when developing interactions incorporating a pressure-based cursor as opposed to, for example, mouse devices or $x-y$ input with a stylus, because the smallest level of pressure that can be distinguished is a much larger percentage of the overall range of input.

Xiaolei [55] performed a study comparing tilt and pressure as input methods for manipulating a one dimensional cursor, finding that tilt was associated with reduced movement time in reaching the target, as well as finding that pressure movement time and error rate had a strong directional effect, i.e. participants found it much easier to 
perform the task when the direction of the target corresponded to an increase in pressure, rather than a decrease.

Bi et al. [5] explored pen rolling as an input method. They were interested in determining a threshold of incidental rolling, beyond which changes in roll could be considered intentional, as well as determining the functional range of roll, in order to determine the maximum size of a roll-based menu that could be comfortably used with a stylus.

Xin et al. [56] ran exploratory studies on tilt as a method of input. They determined ranges of tilt values that could be comfortably used for target acquisition tasks, followed by similar empirical exploration comparing pressure, tilt, and azimuth. They were interested in determining the average natural use profiles (incidental input ranges) of these three input mechanisms, in order to aid in distinguishing intentional gestures using these three types of input.

\subsubsection{Context/grip sensitivity using a stylus device}

Other developments in research on augmented styluses focus on making use of different kinds of hand grips to dynamically infer the interaction that is taking place. Song et al. [40] and Hinckley et al. [22] found that there is a close relationship between how users hold a stylus and their intended purpose, and that users often change grips in relation to the task at hand. The resulting MTPen project and associated work $[40,43]$ made use of capacitive sensing techniques to determine the grip of the user on the stylus. Having determined the user's grip, their system adapted intelligently, changing the mode to suit the task at hand. In Chapter 4, we will return to the concept of grip-dependent interactions using a stylus device; the interactions we designed are also grip-determined. 
The Conté project [50] took another approach with a similar goal: by designing a rectangular device which resembled a Conté crayon, the researchers enabled users to use different tools depending on the side of the device which was touching the touchscreen at any one time.

\subsection{Deformation as an interaction technique}

Still existing almost exclusively in HCI research, deformation is, unlike the stylus, a novel input method. This domain has come to be described under a variety of names, such as DUI (Deformable User Interface) and OUI (Organic User Interface) [49]. Aside from a few exceptions $[38,48]$, the vast majority of work in this domain has focused on interactions occurring on devices that take the form of a slate $[1,29,36,39,42]$. This display-oriented focus is due to recent developments in flexible display technology, and the idea that certain display-related affordances are associated with flexible interactions, such as eliminating occlusion of the fingers on a mobile display [11]. As a result of the specific form factors explored in most prior work on deformable user interfaces, the types of interactions described by our work is fairly unexplored.

Aside from the display-oriented nature of most research, another reason for the particular focus on planar form-factors derives from currently available flex-detection technology. Planar-shaped bend sensors - either thin-film resistive sensors $[7,12,16,29,42,52]$, or planar strain gauges [1,7], are overwhelmingly the most common method of detecting bend interactions in HCI research. These planar sensors can only be flexed along one axis, which makes them ideal for detections with flat-surface formats, but very difficult to use with devices that are designed to detect multiple axes of flex input. Beyond the lack of multiple axes of detection, it is technically challenging to build 
a device using planar sensors that can physically be flexed along a variety of axes. The reasons for these technical limitations will be further outlined in section 3 .

Another distinction regarding prior work with deformable user interfaces is between discrete and continuous input. A number of studies focus on discrete "bend gestures," where the inherent analog input of a flexible device is converted, using thresholding or one-dimensional gesture detection, into an on or off state $[13,16,23,29,30]$. The act of bending a certain area of the device functions as a switch, which can trigger events. This “on-off” classification has been expanded by Warren et al. [52] to include the angle of the axis of bend, as well as the degree of deformation. Given the analog nature of bend input, researchers also have explored flexion as a continuous input method for varying parameters. Since input on the FlexStylus is also inherently analog, this research is somewhat more applicable to our work. Burstyn et al. [7] used bend as a continuous input method for navigating stacked data using a flexible prototype. In-depth studies on the specific ergonomics of flexion as a continuous parameter control were performed by Ahmaniemi et al. [1], Kildal et al. [26], and Burstyn et al. [7]. These studies were all performed on devices with planar form-factors.

It is difficult to gather useful information for the flexible stylus system from the majority of prior research on flexible devices. This is owing to 3 factors: the focus on the deformable device as a surface for displays or facsimiles thereof, the relative dearth of studies that relate to continuous bend input, and the lack of studies that address multiple axes of potential flexion input. There is currently no research which applies deformation detection to a stylus form-factor or anything resembling it. 
However, we have drawn some broad ideas from prior research to inform our work. Within the deformable interface domain, the prior research most relevant to the FlexStylus was performed by Ahmaniemi et al. [1]. This study, which was designed to determine optimal uses of deformation, involved using analog deformation input to control a scrolling task. The researchers found that deformation was a useful input method for tasks that involved continuous, bipolar, input. Because this research was performed using a planar flexible smartphone prototype, it is problematic to generalize its conclusions to a device with a pen form factor. However, certain advantages of using flex input described in that work are equally applicable to non-flat interactions as to flat ones. The most important of these is the deformable device's continuous built-in tactile feedback on the current input state of the device. If the device is bent to a certain extent or at a certain angle, the user will always have feedback on that state because it is an inherent physical property of the device itself.

\subsubsection{Non-Planar Deformation using fibre-optic flex sensing}

While a large majority of prior work in flexible devices has focused on devices with a planar form factor, there are a few examples of flex-sensing devices that are not limited to this form factor. ShapeTape [4] is a notable example of a sophisticated fibre optic deformation and position sensing system (Figure 2). Using this system, users modelled 3D curves using an elongated tool that captures its flexion information in 3D space. However, this device was not intended as a navigation or pointing device, only as a tool for defining 3D curves. 


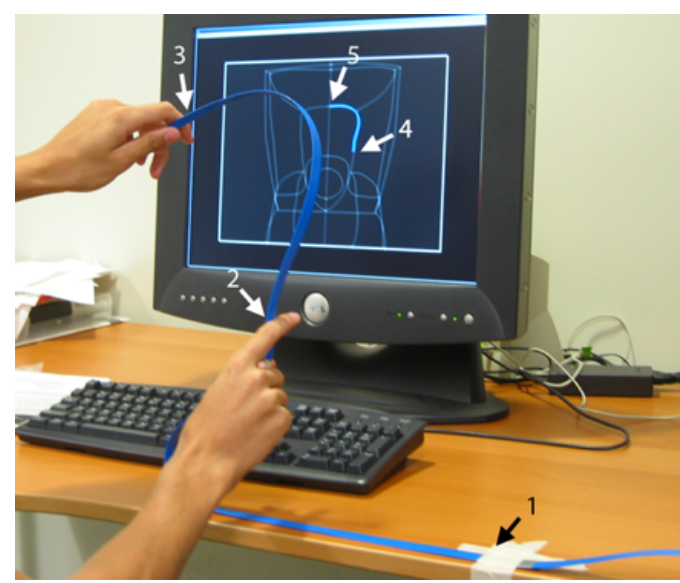

Figure 2: The ShapeTape System [4]

Herkenrath et al. [20] created Twend, a planar, cellphone-sized, deformable interface device that uses a fibre-optic bend sensing technique to detect deformation. The authors envision a device with 8 sensors to detect bend and twist gestures on different areas of the device, and implements a prototype system with 1 unidirectional sensor. There are other examples of fibre-based flex detection in the engineering literature $[14,27]$. These systems are designed for precise architectural applications, and use large and elaborate equipment to measure the deformation of an optical fibre to an extremely high degree of accuracy. Since our device does not have an accuracy-sensing requirement in the order of $10^{-5} \mathrm{~cm}$, and our device has size constraints, this work is not particularly relevant for our design.

\subsection{Other related works}

Previous work on the principle of control-display gain (C/D gain) has informed the analysis of the results of our study (Chapter 7). C/D gain is the relationship between the amplitude of movement of an input device in physical space and the amplitude of movement of the displayed feedback corresponding to that motion. Gibbs [15] used a joystick device to measure the relationship between the distance of travel of an input 
device in real space and the user's targeting performance. Gibbs found that a lower C/D ratio, i.e. larger movement in space of the input device, tended to result in more accuracy. Analysis according to control/display ratio has subsequently been applied to a large number of tasks, from mouse pointing [8] to automotive racing [10]. Since one of the ways in which the flexible stylus differs from a pressure-based stylus is C/D ratio-the very small distance of travel of the pressure-sensitive tip means it will always have a particularly large $\mathrm{C} / \mathrm{D}$ ratio - we propose that $\mathrm{C} / \mathrm{D}$ ratio is one reason for differing performance between the two devices.

Herot and Weinzapfel [21] studied pressure input on a touch sensitive display. They mounted strain gauges on a touch-sensitive display, and used these to measure input from a finger. While this work does not feature a stylus, and - being created in 1978 bears almost no outward similarities to the FlexStylus system, it is possibly the closest relative to our system that can be found in prior work. This is because of the vector nature of the input gathered by the machine (Figure 3). The system detects both input pressure and the direction of that input pressure. It displays both inputs in the form of a vector cursor, which has a length equivalent to pressure, and radiates from the finger at an angle equivalent to the direction of that pressure. This is similar to the resulting output of the FlexStylus, a vector that combines the magnitude of the flex input and its angle.
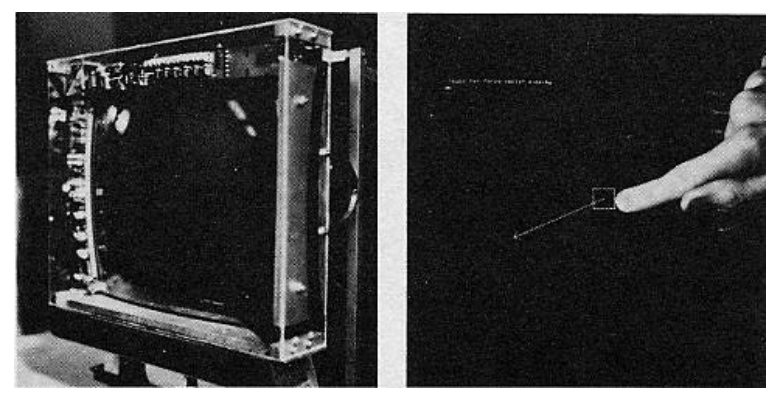

Figure 3: Herot and Weinzapfel's pressure sensitive display [21], with force vector 


\section{Chapter: FlexStylus Prototype}

\subsection{Limitations of currently used flex sensing technologies}

Most deformable user interface prototypes [29,42,46,48] use thin-film bend sensing technologies. Thin film sensors use a "sandwich" made up of two high conductivity strips enclosing a strip with a pressure-based variable resistance (Error! Reference source not found.). As they are deformed, the inherent stress of the deformation causes force to be applied to the pressure sensitive strip. As a result, the net resistance changes, which can be measured using a voltage divider. These sensors are useful for planar-shaped prototype devices, but it is only possible to bend them in certain directions - they can be twisted or curled, but not deformed side-to-side. This limits the form-factor of potential prototypes to configurations that only bend on one axis.

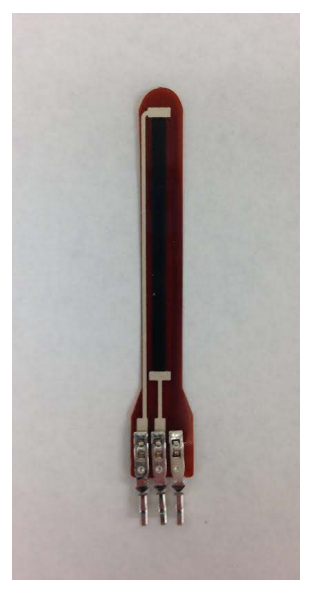

Figure 4: FlexPoint resistive bend sensor

While such configurations are useful for studying planar prototype devices, they limit the ability to explore types of bend interactions that are not on planar surfaces, and use of these sensors also disqualifies the study of interactions which make use of more than one axis of bend control. A notable exception to this is the Twend prototype [20], which uses fibre-optic detection (Figure 5). The fibre-optic flex technique used in the 
Twend device informed the construction of the FlexStylus prototype. However, the Twend system uses one filament as opposed to the FlexStylus' 4, and therefore has different flex sensing capabilities from the multi-filament technique used in the FlexStylus.

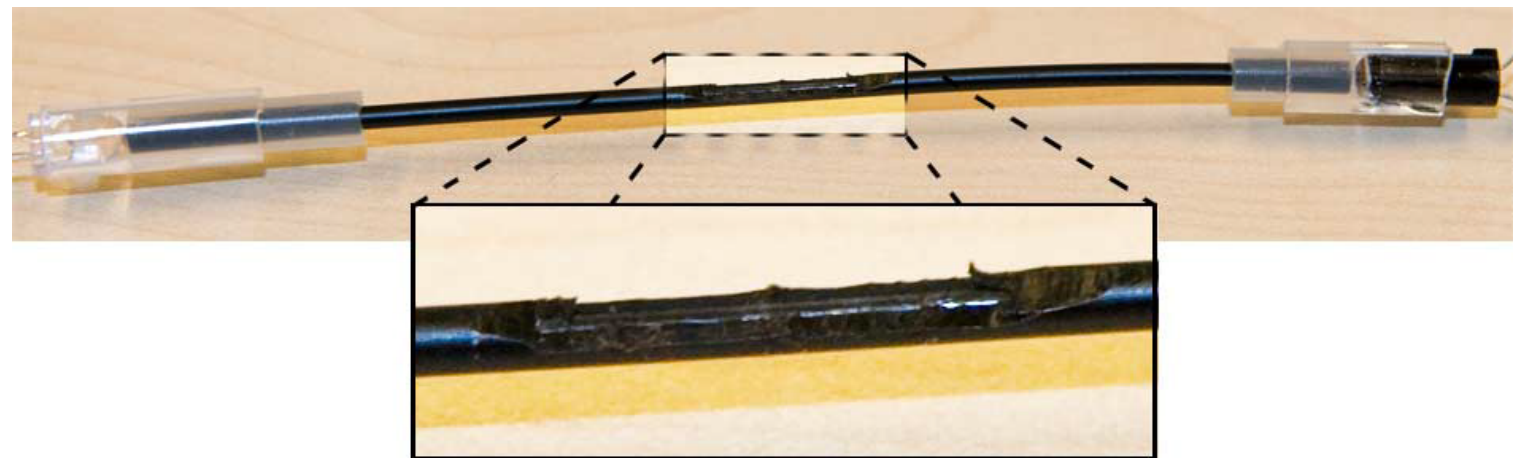

Figure 5: Fibre-optic Sensor from Twend prototype, showing abrasion

\subsection{Outline of flex sensing technique for flexible stylus}

The FlexStylus device uses a cluster of 4 fibre-optic cables, coupled on one end to a shared 3mm infrared light-emitting diode (IR LED). At the base of the stylus, each fibre-optic cable is mounted to a surface mount (SMD) phototransistor cell, which generates current based on luminescent input. This signal is read by an Arduino Uno [3]microcontroller using four 8-bit ADCs (Analog-Digital Converters), and registered in the software system. The resulting four values can be used to calculate several useful data points about the configuration of the pen. The two most useful resulting values are: an absolute value of deformation (Figure 6), which indicates in absolute terms how far the device is flexed, and a value representing the angle of deformation--the angle in reference to a fixed point at which this flexion is taking place (Figure 6). 


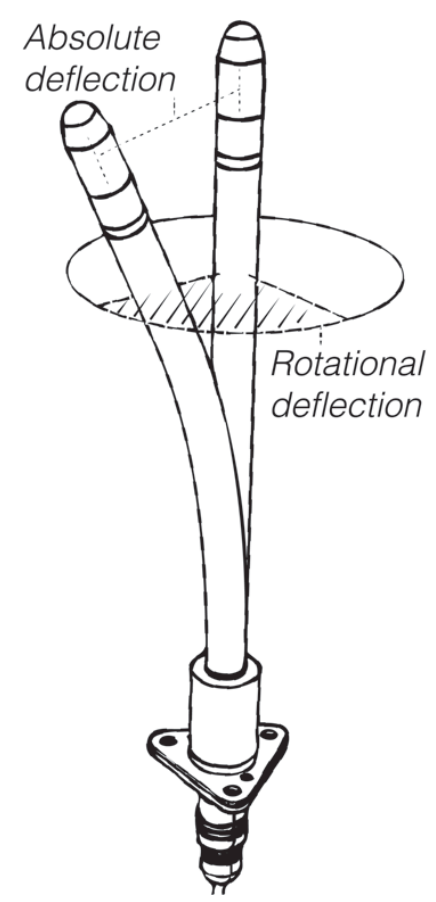

Figure 6: Degrees of deformation freedom detected by FlexStylus

\subsubsection{Mechanism of operation}

We selectively abrade each fibre-optic cable to enable it to detect deformation. In the initial two prototypes, this was performed by hand using a razor blade, while for the final prototype, we designed a 3D-printed nylon jig to improve precision and reduce build time. When assembling the device, we align these abrasions at $90^{\circ}$ to one another within the cluster of four cables, so that each abraded side faces directly outwards. As a result, the transmissive properties of the fibre-optic cable become dependent on the flex configuration of the device. If the cable is flexed towards the abraded portion of the fibreoptic cable, a percentage of the light escapes the cable in proportion to the degree of flexion (Figure 7). Conversely, when the fibre is flexed away from the abraded portion, more light is retained in the fibre. The overall amount of light retained in the fibre is read by phototransistors, and is converted into a voltage (Figure 8). 


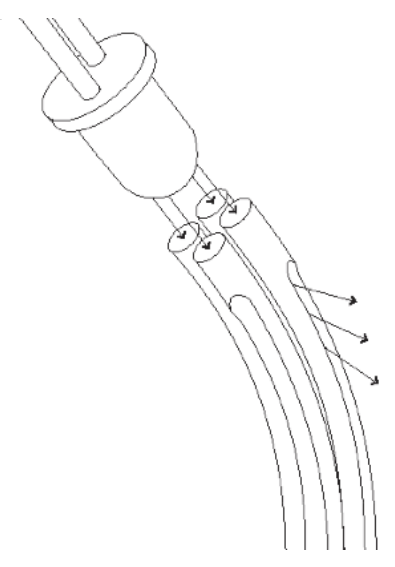

Figure 7: Mechanism of operation of flex sensor cluster

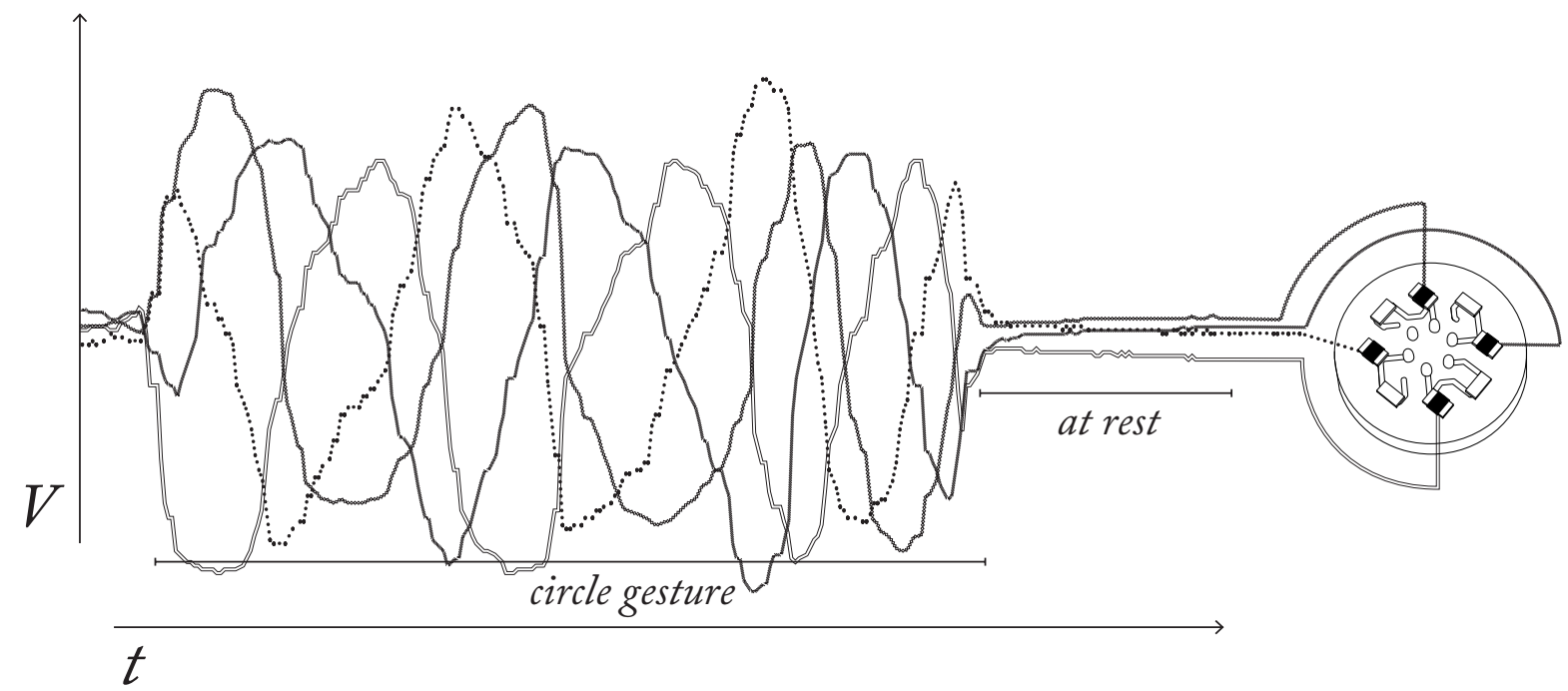

Figure 8: Relative voltage outputs of bend sensors during circle gesture, showing PCB with phototransistors (right)

In order to maximize light transfer from the IR LED into the ends of the fibres and from the base of the fibre sensors to the phototransistor array, we smoothed the ends of the fibre-optic cables to a highly smooth finish. This involved buffing the tips of the fibres by hand using silicon carbide abrasive film. We employed a sequence of abrasive films with decreasing grit sizes, from 15 microns, to 5 microns, to 0.5 microns. 


\subsection{Design challenges in prototype construction}

The primary limitation in the design and construction of the prototype was size. Because it is intended to be used as a stylus device, it was important to create a device with a size and weight similar to an ordinary stylus. Research on ballpoint pens has showed a negative correlation between size and drawing accuracy, with a circular diameter of $8 \mathrm{~mm}$ being favoured over pens with a 15 or $20 \mathrm{~mm}$ diameter [17]. We designed the device to have a cross-sectional diameter as close as possible to the ideal described by this study, with the final prototype having a cross sectional diameter of 9 $\mathrm{mm}$ at the thickest point along the shaft. Also, because the study would be comparing the device to a commercial stylus device, it was important to make the best possible effort to match the properties of a commercial stylus. We therefore made choices to favour compactness in designing and fabricating the prototype. For example, we created a custom PCB to hold the phototransistors, in order to minimize size and weight.

\subsection{Iterative construction of prototype}

This section will include an in depth discussion of the manner of construction and operation of the prototype stylus device used for testing.

\subsubsection{Non-functional mockups, interaction sketching, and sensor proof of concept}

We explored ideas of the form of the prototype through non-operational clay mockups (Figure 9, right). These allowed us to brainstorm and explore the feasibility of performing various interactions with the flexible stylus, and informed future decisions regarding which interactions to pursue. We also created a series of sketches to illustrate a variety of potential interaction techniques (Figure 9, left). 

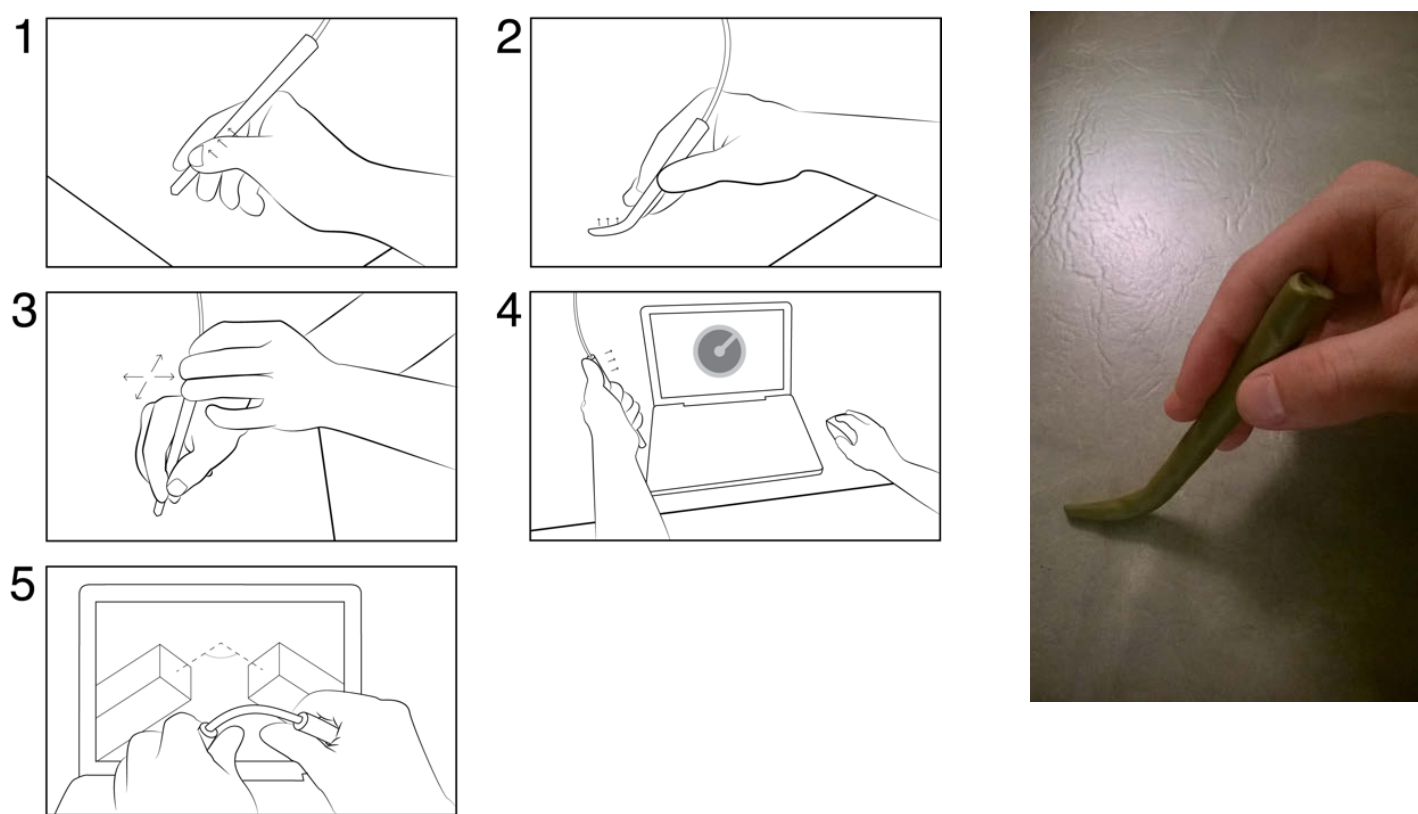

Figure 9: Initial sketches (left) and clay mockup prototype (right)

After having determined that optical flexion detection would be an ideal approach for creating a device possessing the required qualities for the project, we first created a proof-of-concept prototype to test the sensing mechanism. The first prototype was a rudimentary one. It consisted of a single, $2 \mathrm{~mm}$ diameter, plastic optical fibre (POF). On one end of the device we attached a 5mm white LED, and on the other end of the device we attached a through-hole photoresistor. The $2 \mathrm{~mm}$ POF was abraded asymmetrically, then encased in a heat-shrink tubing, in order to prevent incidental light from being transmitted to the fibre through the abrasion. The LED and photoresistor were coupled to the optical fibre using larger diameter heat shrink tubing. The resistance of the photoresistor was measured using a simple voltage divider and connected to an Arduino Uno ADC pin. The output of this initial experimentation proved surprisingly stable, and, importantly, this initial prototype only registered bends in one direction. 


\subsubsection{First FlexStylus Prototype}

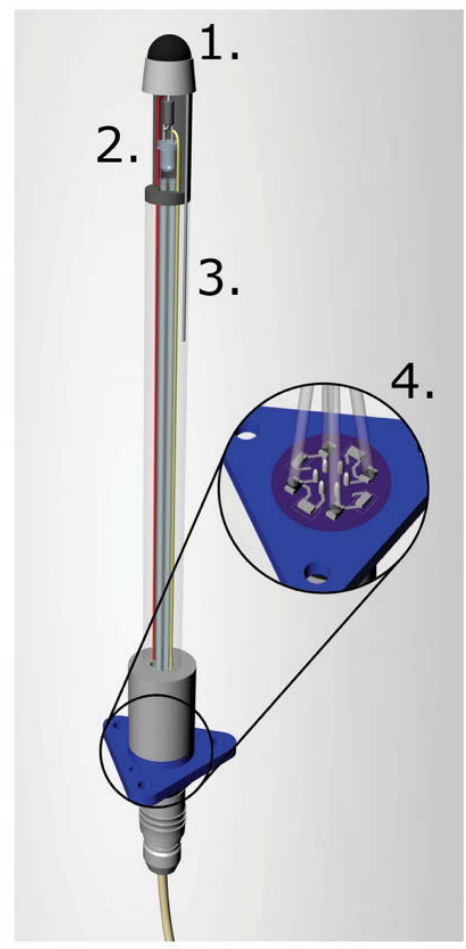

Figure 10: Cutaway of First Flexstylus prototype

The first version of the FlexStylus (Figure 10, Figure 11) was designed to implement the "cluster" method of multiple flex sensors, in order to test preliminary interactions using the flexible stylus device. We created a round circuit board $($ diameter $=$ $14 \mathrm{~mm}$ ), using EAGLE CAD. This circuit board included the necessary electronics for implementing a luminescent sensing circuit (Figure 10, \#4). The phototransistors (Figure 10, \#4, were manufactured by Kingbright (model APT2012P3BT) and the resistors were $10 \mathrm{~K} \Omega \mathrm{SMD}$ resistors. This resistance value was chosen to create a range of voltage outputs that were within the Arduino's Uno's ADC detection range (5V). The phototransistors were connected to the resistors and outputs with a simple voltage divider circuit. The circuit boards had through-hole connector inputs to the 6-pin Hirose HR10 micro connector. Four of the 6 pins were used to convey the analog information from the 
phototransistors back to the Arduino, and the other two pins provided power (5V) and ground from the Arduino to the phototransistor circuits. These pins were connected to wires that travelled through the device (Figure 10,\#3) functioning as power and ground lines also for the $940 \mathrm{~nm}$ IR LED (Figure 10, \#2) mounted at the tip of the device. We mounted a conductive rubber tip (Figure 10,\#1) to the stylus to enable $x-y$ input on capacitive touch screens.

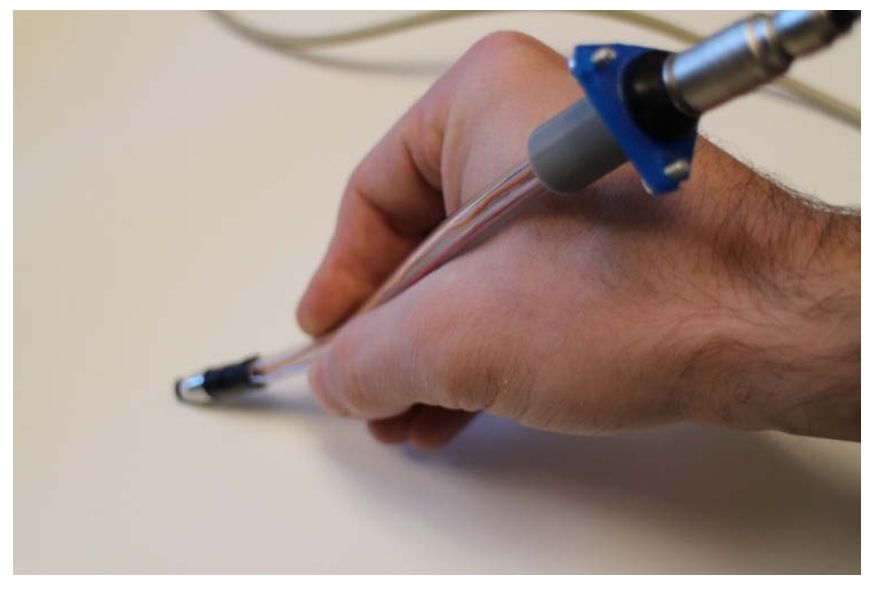

Figure 11: Photo of First Flexstylus prototype

One of the challenges in building this prototype was that the coupling between cables and phototransistors needed to be very accurate, and due to the overall pen-shape of the device, there was a limited amount of space to work with. In order to create a physical prototype that obeyed the design's constraints of small size and accurate coupling, an iterative process involving the 3D printing of several CAD designs was necessary. We designed all components using the Rhinoceros 3D editor, converted to stereolithography (.STL) files, and 3D printed using a Makerbot 2 PLA printer.

\subsubsection{Final Prototype}

We designed the next prototype to be more modular, allowing the connection of multiple kinds of input processing to the flexion sensors, as well as the possibility of 
reconnecting or changing tips. The main goal of this modularity was to create a prototype system which could be easily extended and repaired. We preserved the general electronics layout from the initial prototype, however, we redesigned the plastic housing. There were three prominent changes to the design of the prototype: the removable tip, the extended rigid plastic section of the body, and the prototype's method of fabrication.

\subsubsection{Removable tip}

The final prototype of the flexible stylus incorporated a removable tip (Figure 12). The removable tip facilitated the construction of the device, allowing the device to be created in two separate modules. One of the largest challenges involved in the construction of the first prototype was routing the power wires for the LED through the body of the pen. These had to be threaded through a small plastic coupling piece, and once soldered into place, it was impossible to take the device apart for examination or repair. Using a detachable tip, the tip could be constructed at the same time as the rest of the device, and the two components could be tested separately from one another, which made troubleshooting the device much simpler. The removable tip also enabled extensibility. In the interest of future research projects, which may look to include more types of augmentation (such as a passive magnetic position sensor, similar to the Microsoft N-Trig system), it would be preferable to have an easily interchangeable tip.

We constructed the removable tip with the aid of small machine pins with their plastic housing removed. These enabled the LED power to be transmitted from the shaft of the stylus device into the removable tip. The friction of these pins helps to hold the removable tip on the end of the FlexStylus. The tip was also held in place through the tight coupling between the alignment piece, which holds the fibre-optic cables in place, 
and the removeable tip, which overlaps the main body of the stylus when the two are affixed together. For user studies, the segment of the device connecting the removable tip to the body was reinforced with self-adhesive tape, to ensure robustness.

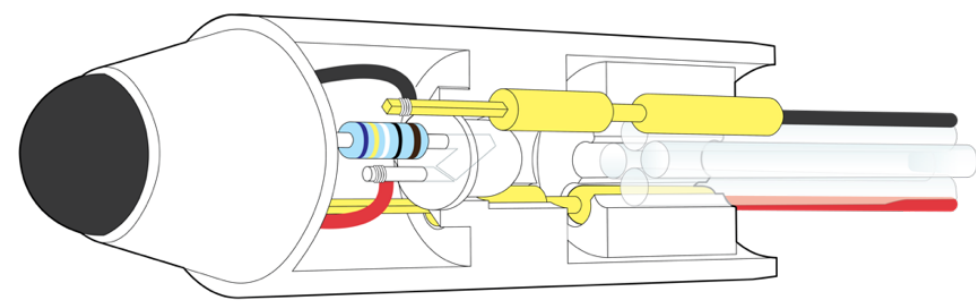

Figure 12: Cutaway of Removable Tip

\subsubsection{Extended Rigid Stylus Body}

The design decision which had the largest visible effect on the final stylus revision is the incorporation of a longer rigid section to the body of the device (Figure 13). Both iterations of the prototype are approximately $18 \mathrm{~cm}$ in length. In the case of the initial prototype, the majority of the device, approximately $15 \mathrm{~cm}$, was composed of a flexible tube. In the new prototype, the flexible portion of the device was $5 \mathrm{~cm}$ long, owing to the rigid base, as well as the slightly longer rigid tip. Despite the visible difference, this decision had little effect on the performance of the device. We noted with the original prototype that the majority of deformation took place in the $1 / 3$ (approximately $6 \mathrm{~cm}$ ) closest to the tip of the device. This is the portion of the device that it is possible to flex while the device is held in a pen grip. Even in the case of varying grips, it is not difficult to flex the prototype with the elongated rigid section, because the force is transmitted to the flexible portion of the device. There were two advantages to 
deciding to incorporate a longer rigid section in the base of the device. The first was to reduce unwanted flexion caused by the weight of the phototransistor portion pulling downwards. The second reason was robustness: a shorter flexible section, while it reduced the active flexible area, also reduced the amount of strain on the components of the device.

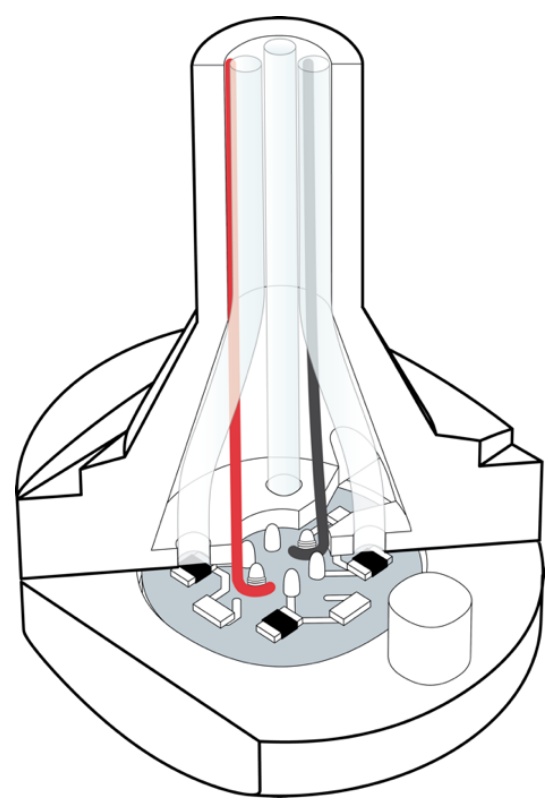

Figure 13: Final prototype body design, showing phototransistor circuit

\subsubsection{Device Construction}

Because the new methods of construction introduced a certain amount of complexity into the construction of the device, in particular to the internal geometry of the system, it was necessary to fabricate the device using more sophisticated technologies. The parts were, once again, designed in Rhinoceros 3D, but rather than being fabricated using a PLA extrusion system as before, the 3D models were printed by an external company (Sculpteo). The phototransistor portion at the base of the device was constructed in two modules, which were aligned using a hole and peg system (Figure 13). 
The principle of this choice was to decouple the phototransistor section from the stylus itself, to enable potential future replacement with improved hardware, such as a microcontroller with wireless capabilities. 


\section{Chapter: Interaction Techniques}

\subsection{Hand grips and context}

While many input devices, such as a mouse devices, joysticks, or non-augmented styluses, can be gripped in various ways, the way that they are gripped does not have a strong effect on the functional degrees of freedom of the device. This is not true for the FlexStylus; a user's ability to provide input to the FlexStylus is constrained by the grip they employ to hold it. Because possible degrees of input freedom of the flexible stylus are highly grip-dependent, grip is of considerable importance to the design of interaction techniques. Therefore, interactions with the FlexStylus must be designed to operate within the specific biomechanical strengths and limitations of the hand depending on the grip context. There are a number of different ways that a user might decide to hold a stylus. We do not intend to present an exhaustive list of potential grips, but more a series of categories which are clearly connected to different types of interactions on the FlexStylus prototype. There is nothing to prevent the user from holding the stylus any way that they would like, but we have designed interactions to make use of particular clear affordances of different ways of holding the device. Thus far, we have implemented interaction techniques based on three grip types: the tool grip, the menu grip, and the scroll grip (Figure 14).

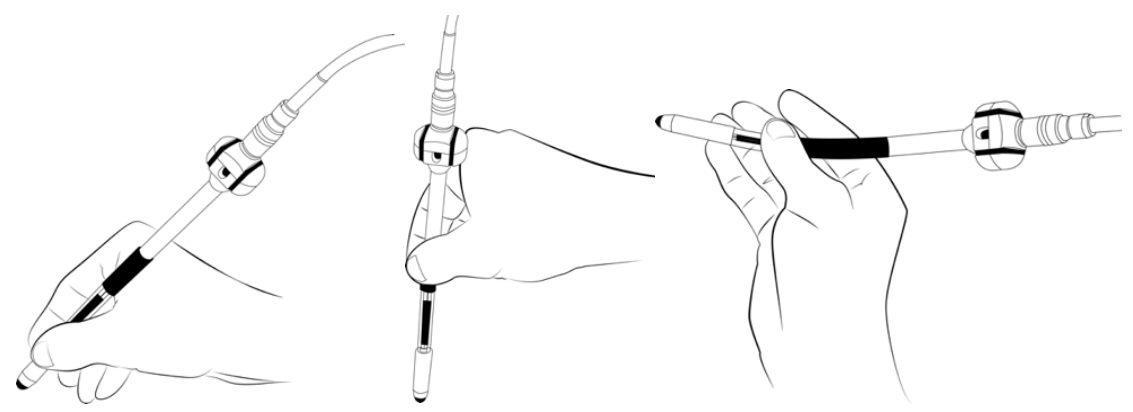

Figure 14: (left to right) Tool Grip, Menu Grip, Scroll Grip 
In these three grips, there is a relationship between the breadth of the possible angular flexion input, and the amount of $x-y$ input possible. The tool grip offers the most control over x-y input, but the least control over the angle and magnitude of bend input. The menu grip is associated with fixed $x-y$ position, but users have a wider amount of control over the angle of flexion. The scroll grip allows users to have control over both angle and amount of flexion, but does not allow for $\mathrm{x}-\mathrm{y}$ input.

We exploit the properties of the grip explained above by designing interactions that accommodate the degrees of input freedom associated with each grip. The menu grip provides the most control using bend, and is therefore the basis for a global pie menutype widget. A tool-specific context menu, with fewer options, is associated with the tool grip, which has a smaller potential range of motion than the menu grip. This allows users to make tool option choices without having to change their grip on the pen. The scrolling grip occurs when the pen is lifted from the tablet, so we associate this grip with navigation, rather than input.

\subsection{The Tool Grip: Drawing and Context Menus}

\subsubsection{Augmented Drawing}

Digital drawing uses several continuous parameters, such as brush size, hue, light, saturation and transparency. With a basic stylus, the user cannot control any of these parameters while drawing. Most drawing tablets augment this basic functionality using pressure on the tip of the device to control one parameter. Deformation can be used to control one or two of these parameters (Figure 15). Previous studies showed that users can control both pressure and tilt [19] or grip [45] simultaneously while drawing. We 
intend to use the FlexStylus prototype to evaluate the exact extent of users' abilities to control the angle of deformation while holding the pen in the tool grip in our future work.

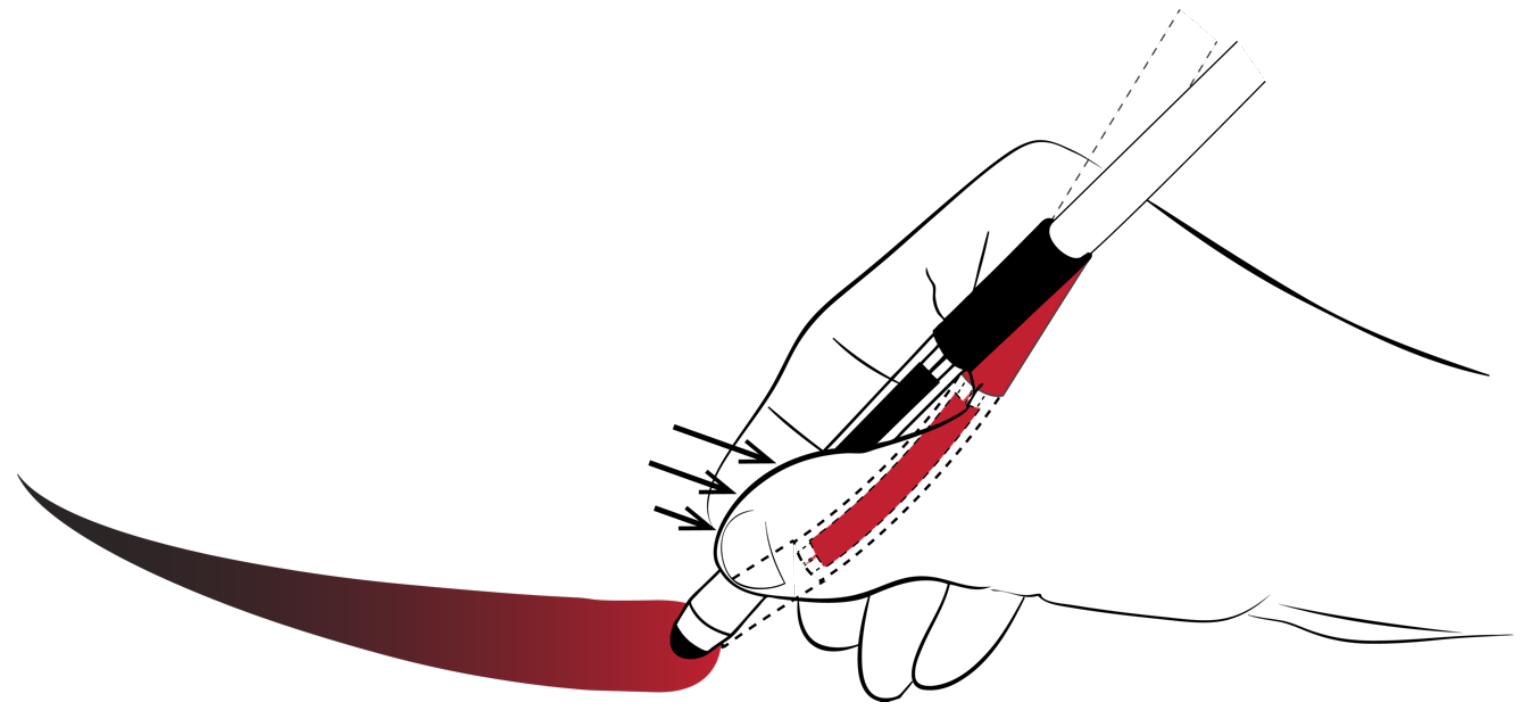

Figure 15: Using deformation to control stroke width

\subsubsection{Context Menus While Drawing}

In a typical drawing application, the user selects a brush shape and size, as well as a color. When they would like to change one of these parameters, they must select another value in the appropriate toolbar. Alternative toolbars such as Toolglasses [6] avoid round trips between the drawing area and the toolbar. However, these alternate toolbars require either another pointing device or a mode switcher to avoid cursor movements. FlexStylus offers a secondary pointing method using a single one-handed device.

When drawing, the user grips the stylus like a pen to easily control the tip position on the tablet. The range of possible angular bends is restricted when using the tool grip. Rather than being able to rotate the device freely, the user is limited to applying force on the device using one finger at a time, while the device is being supported by the other 
fingers. While holding the FlexStylus in the most common type of pen grip, the tripod grip $[25,40]$, users can supply continuous input using either the thumb or the index finger. Context menus for use while holding the device with the tool grip must be designed to accommodate these limitations. However, two parameters of continuously variable input are still adequate for menu selection tasks, especially given the smaller menu sizes associated with contextual tool menus.

For these contexts, we propose a menu consisting of L-shaped gestures. Pressure on the $\mathrm{x}$-axis of the device (i.e., the thumb in the conventional tripod grip) allows the maneuvering of the cursor over various menu items, while pressure on the device in the y-axis (i.e., the index finger), allows for the selection of those menu items. Furthermore, since this y parameter is a continuously variable value, users can use the degree of flexion to make parametric selections within the menu item. For example, the user uses thumb pressure to cycle through the context menu, eventually finding the brush size modifier. Then, the brush size can be selected with precision using pressure from the index finger.

\subsection{The Menu Grip: Menus and Selections}

The user can also grip FlexStylus by holding the rigid section above the flexible one. This "menu grip" is useful because it does not present a large variance in the hand's ability to select items based on direction, giving the user the opportunity to make use of the full range of angular degrees of freedom. The hand is equally capable of moving in the four directions, and provides deformation input using motions similar to using a large arcade joystick. The tip of the pen is the fixed point against which the pen bends, statically posed on the screen through frictional force and slight down-pressure. 


\subsubsection{Marking Menus}

The menu grip enables interaction with menus, such as radial or marking menus [28]. The user selects the marking menu element by angling the FlexStylus in the direction of the element. The user selects the menu item by bending the device past a certain bend threshold. Since these menus operate using the same principles as marking menus, we theorize that the same advantages apply - for example, as users become more experienced, the menu can be removed. Thus far, we have only prototyped one level of radial menu, but as a result of the gesture input recognition capabilities of the FlexStylus (see section 4.3.3), multiple levels are possible. Future work will involve prototyping multi-level marking menus, and performing a study on the learnability of marking gestures using flexion as input compared to other means of pointing input. Figure 16 illustrates a radial menu.

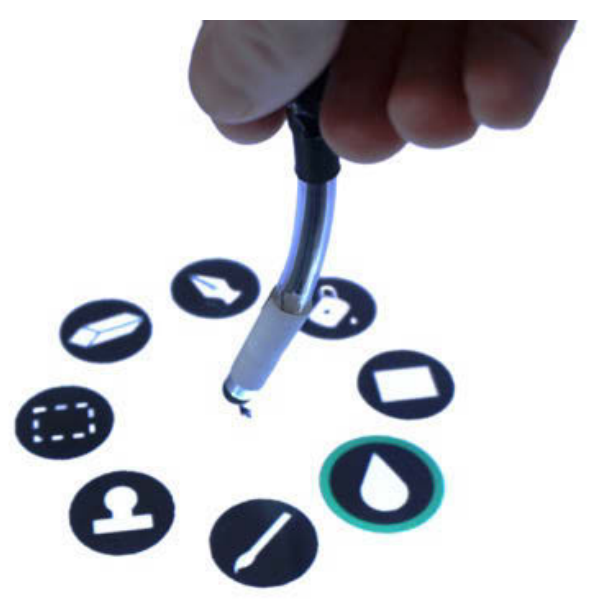

Figure 16: Selecting Drawing tools with Radial Menu

\subsubsection{Selection Interactions}

We added a button to the FlexStylus prototype (Figure 1) to enable selection interactions where crossing gestures cannot be used. For instance, this is used in menus 
that do not offer a series of discrete options, but instead a continuum of possible selections. An example of one such menu is a 2 dimensional colour wheel, where the angle of the bend determines colour hue, and the degree of bend determines colour saturation (Figure 17). Since the precise position of the stylus is important, the button is employed to allow for selection operations without changing the bend state of the stylus. It is worth noting, however, that adding a button is not ideal; the force required to depress the button causes the amount of deformation to change slightly, which is detrimental for precise selection. We propose an alternative in the form of a capacitive touch sensor, which would allow the user to lightly touch the device to make selections without changing the device's bend state. This, however, has not yet been implemented.

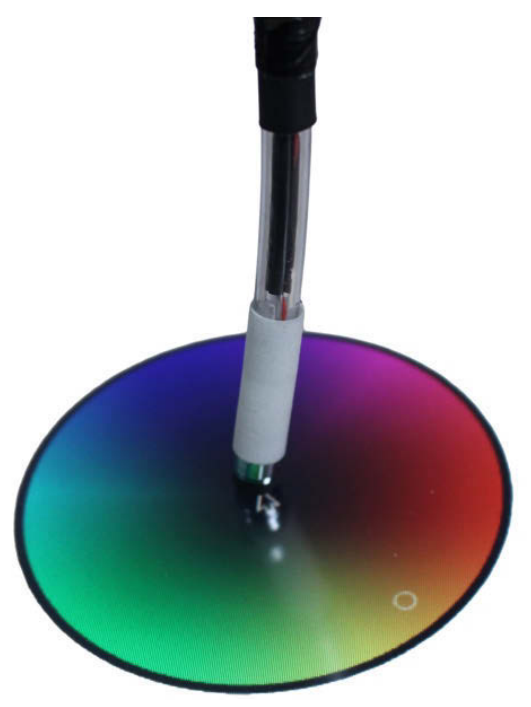

Figure 17: Selecting from a continuous spectrum with the menu grip

\subsubsection{Gesture input}

We were able to use the secondary pointer created by the combination of bend angle and amplitude to interpret gestures, using the $1 \$$ Unistroke Recognizer [54]. We propose two uses for gesture detection. One pertains to state changes (described in 
section 4.1). We specify a particular shape gesture that, due to the limitations of flexion input with other grips, indicates that the user is holding the stylus in the menu grip. Also, shape gestures can be used to select specific commands, similar to gestures with any other pointing device. In the context of drawing, we suggest that primitive shapes be used for drawing those same shapes, i.e., users can select the circle tool by bending the device such that the secondary pointer describes a circle (Figure 18)

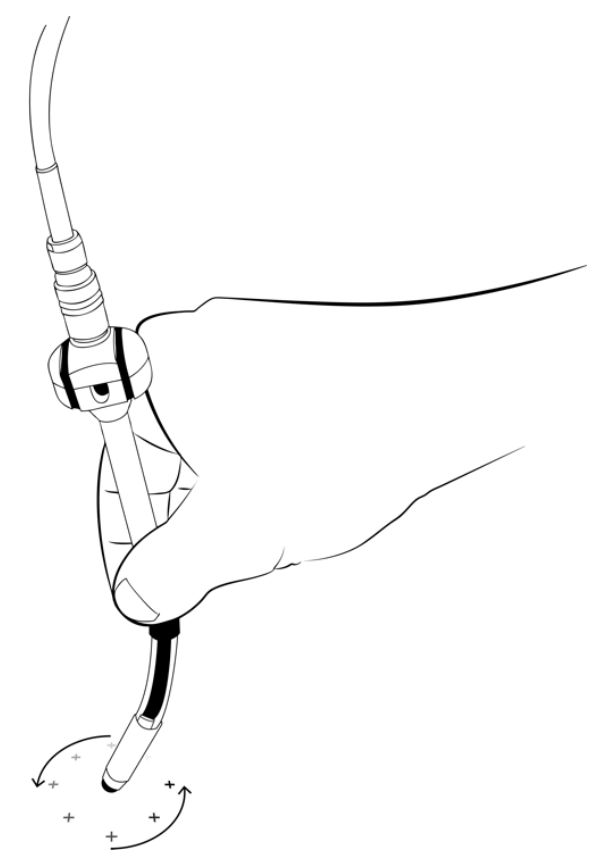

Figure 18: Circle gesture

\subsection{Gesture for transition from tool to menu grip}

One of the utilities of gestural input is that it provides the system with a limited ability to infer the user's current grip from gestural input. We propose using a specific gesture to transition from the tool grip mode into the fully circular marking menu associated with menu grip mode. This gesture limits its own use by being very difficult to perform while holding the device with the tool grip. It is thus a method of seamlessly ensuring that when users summon the radial menu, they are holding the device in way 
that allows them to make use of its full functionality. We acknowledge that users will hold the device in their own idiosyncratic ways. Whatever way the user is holding the device, if they are able to perform a gesture that encapsulates the range of angles needed to use the radial menu, they are also able to make use of the full functionality of that menu.

\subsection{The Scroll grip: Mid-Air interactions}

The system enters the state corresponding to a scroll grip when the user removes the stylus from the tablet, yet continues to provide deformation input. Removing the stylus from the tablet limits the kinds of grips possible: for instance, the menu grip cannot be used because the user is not able to use the friction of the surface to provide the counterforce necessary to bend the device precisely. However, it enables the scroll grip; by balancing the bend force between the two fingers, and pressing with the thumb, the user can roll the device between their fingers.

These in-air interactions are ideally suited for navigation operations, as navigation operates using relative motion more than through direct mapping in the way that a pen tool would. This squeeze and roll technique can be used in any situation where there are two linear directions as well as an absolute magnitude. We propose using the change in bend rotation (the angular motion's direction) to determine the direction of unidirectional scrolling (i.e. to scroll up and down a document), while the size of the bend can be used to control scrolling rate. The scrolling interaction can be extended to include any kind of similar navigation, such as scrubbing. For example: in the context of a drawing program, a user could use this type of operation to cycle through an animation, or through different paintbrush tips. 


\section{Chapter: Experiment}

While we have proposed a selection of potential interaction techniques for the FlexStylus, these are highly context-specific. Because this is the first experiment to be conducted on a device of this type, we chose to begin with a study that examines users' interactions with the FlexStylus in more general terms. To this end, we focused on a particular scenario: providing continuous input to the device, as in the case of modifying stroke width during drawing. The structuring metaphor for the user study can be thought of as a user employing the stylus for writing a solid line on the digital page, where the thickness of the line is dependent on the absolute amount of flexion in the device (see section 4.2.1 for a discussion of augmented drawing).

Although it is helpful to think about this experiment in terms of matching a stroke, the experiment is also intended to provide information regarding users' ability to provide precise input in general. For this reason, we consider that the findings will be not only applicable to situations where continuous input is necessary (such as modifying a brushstroke), but any situation where a user makes use of absolute flexion to provide input in some way. These interaction cases might include menu selections, scrolling, camera, or viewport movement.

We chose to look at only one of the two possible degrees of freedom of the flexible stylus: absolute flexion. We chose to focus the efforts of the study on this absolute flexion because it is most similar to the most commonly used pre-existing stylus augmentation - pressure. Both absolute flexion and pressure are analog input values defined as a distance from a neutral resting state. There is no analogous input for angular flexion input with a typical pressure-augmented stylus. Future work will include 
comparing angular flex input with a tilt-based augmented stylus, the closest alreadyexisting relative to that kind of input. We compared the performance of the FlexStylus against that of a standard commercial pressure-sensitive stylus from a Microsoft Surface Pro 2 [31].

\subsection{Description of task}

We performed a $2 \times 3 \times 3$ study with 2 types of stylus (flexible stylus or pressure stylus), 3 types of target function (square, sine, triangle) and 3 function sizes (small, medium, large). Our dependent variable was accuracy, measured as the absolute difference between the user's input and the target, in pixels $(1 \mathrm{px}=0.12 \mathrm{~mm})$.

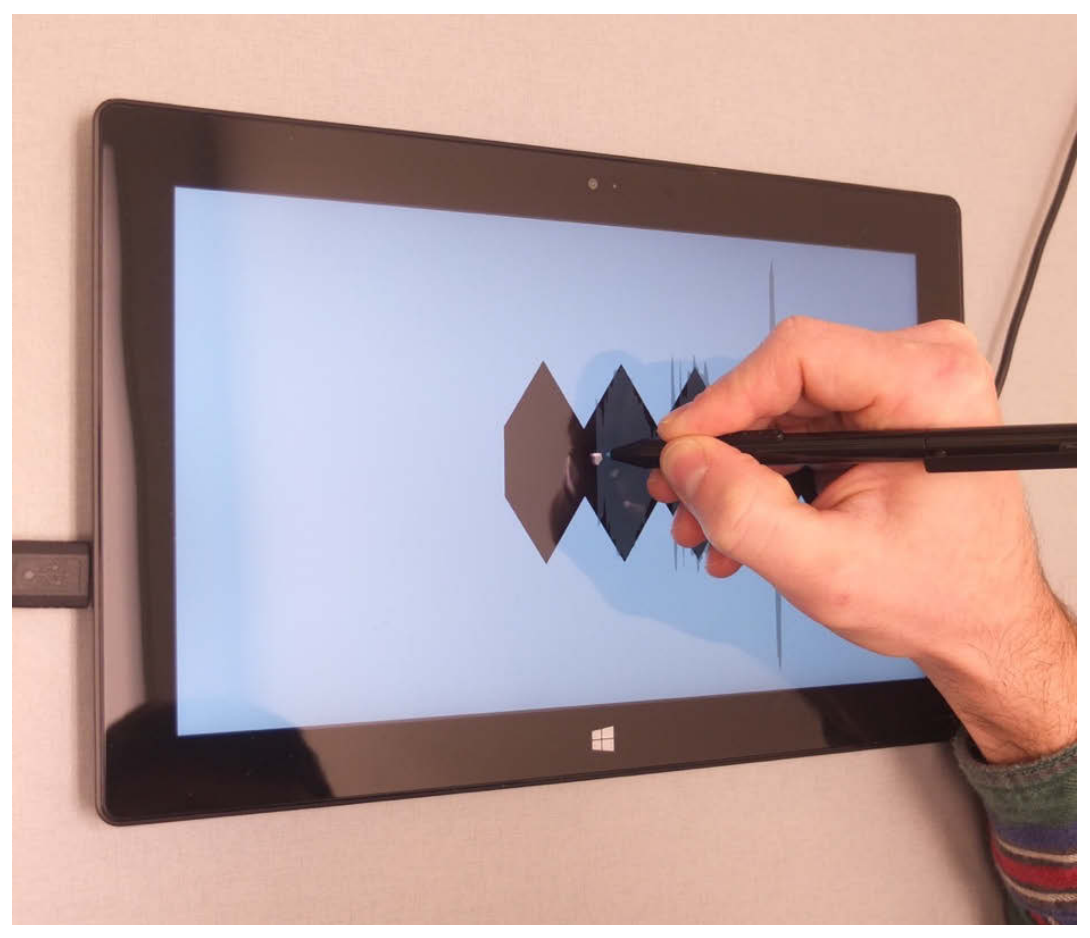

Figure 19: Study Apparatus, pressure stylus trial, triangular curve

Participants were asked to modulate the stylus input to match a scrolling target function (see section 5.3 for detailed description of functions), while holding the input device still in approximately the centre of the screen (though this was not formally 
constrained). The input was either pressure, under the pressure stylus input device condition, or absolute deformation, under the FlexStylus input type condition.
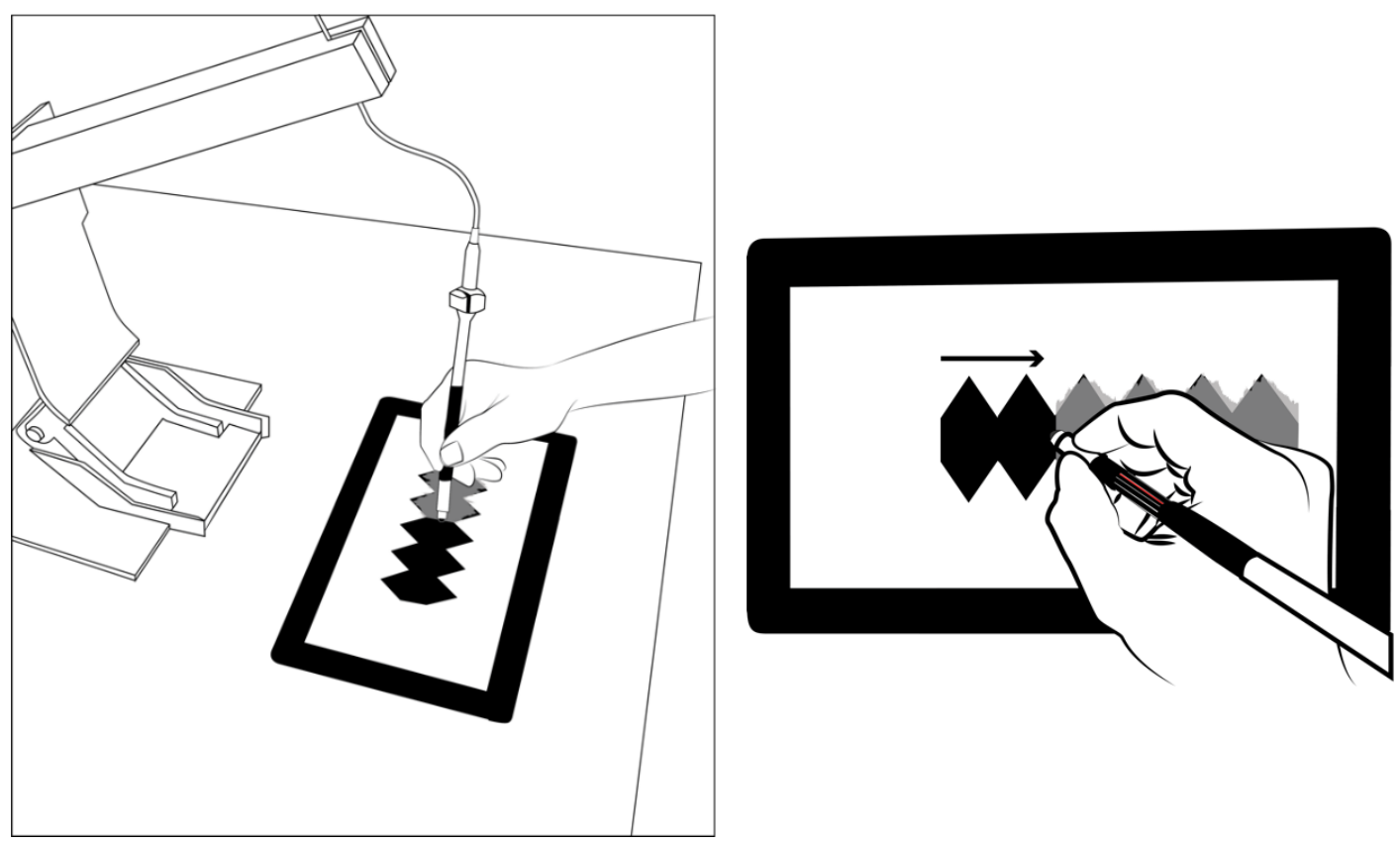

Figure 20: Study setup (left), study detail (right) Arrow indicates function scrolling direction

Visual feedback consisted of a grey function that extended rightward from the stylus as time progressed. The resulting feedback was a shape representing the user's input over time, superimposed over the target function. The input feedback was overlaid on the target using transparency so that participants could easily see the target regardless of input. The participant's objective was to modulate input in response to the target function in a way that produced a feedback function that matched, as closely as possible, the input function.

The upper and lower halves of the screen were mirror images of one another. The purpose of this mirroring was to make the experiment more closely resemble a use-case where the user varies stroke width, with the stylus in the centre of the stroke, and the 
width being determined by either pressure or deformation. For simplicity, we will describe target and input values as distances from centre of the screen.

The x-y position of the stylus was not recorded, because we were interested in only studying pressure or flex input. Participants' grip was not formally constrained, aside from the suggestion that participants hold the device in a comfortable way that they could write or draw with. While participants used a variety of grips, they would all be classified as falling under the "tool grip" category of our grip classification system (see section 4.1). To avoid the weight of the FlexStylus cable interfering with observed deformation measurements, it was supported above the stylus with a stand (see Figure 20).

\subsection{Protocol}

After signing consent (Appendix A.6) and participant information (Appendix A.1) forms, we asked participants to sit at the experimental system, a Microsoft Surface Pro 2. We explained the task (see 5.1 for detailed description of task), and performed a brief demonstration of the task. Condition order was pre-selected using a randomized Latin square. Half of the participants started with the pressure stylus, and performed all combinations of functions and sizes, followed by all combinations with the flexible stylus. The other half of participants began with the flexible stylus.

After completing the tasks with one input type, we provided participants with a feedback questionnaire with Likert-style responses (see Appendix A.2 and A.3). They completed a similar questionnaire following all trials with the second input type. The questionnaire accompanying FlexStylus trials also included feedback questions on the dimensions and physical qualities of the prototype. After having completed both 
segments of the task, participants answered a questionnaire that contained comparison questions regarding which stylus they felt was more accurate, more comfortable, and which they preferred overall. In total, the experiment took approximately one hour.

\subsection{Target Functions}

We chose to study 3 functions as targets. Each function was associated with 3 amplitudes. Anticipating that flexion and pressure may have different strengths and weaknesses depending on context, we chose the functions in order to study specific aspects of pen movement.
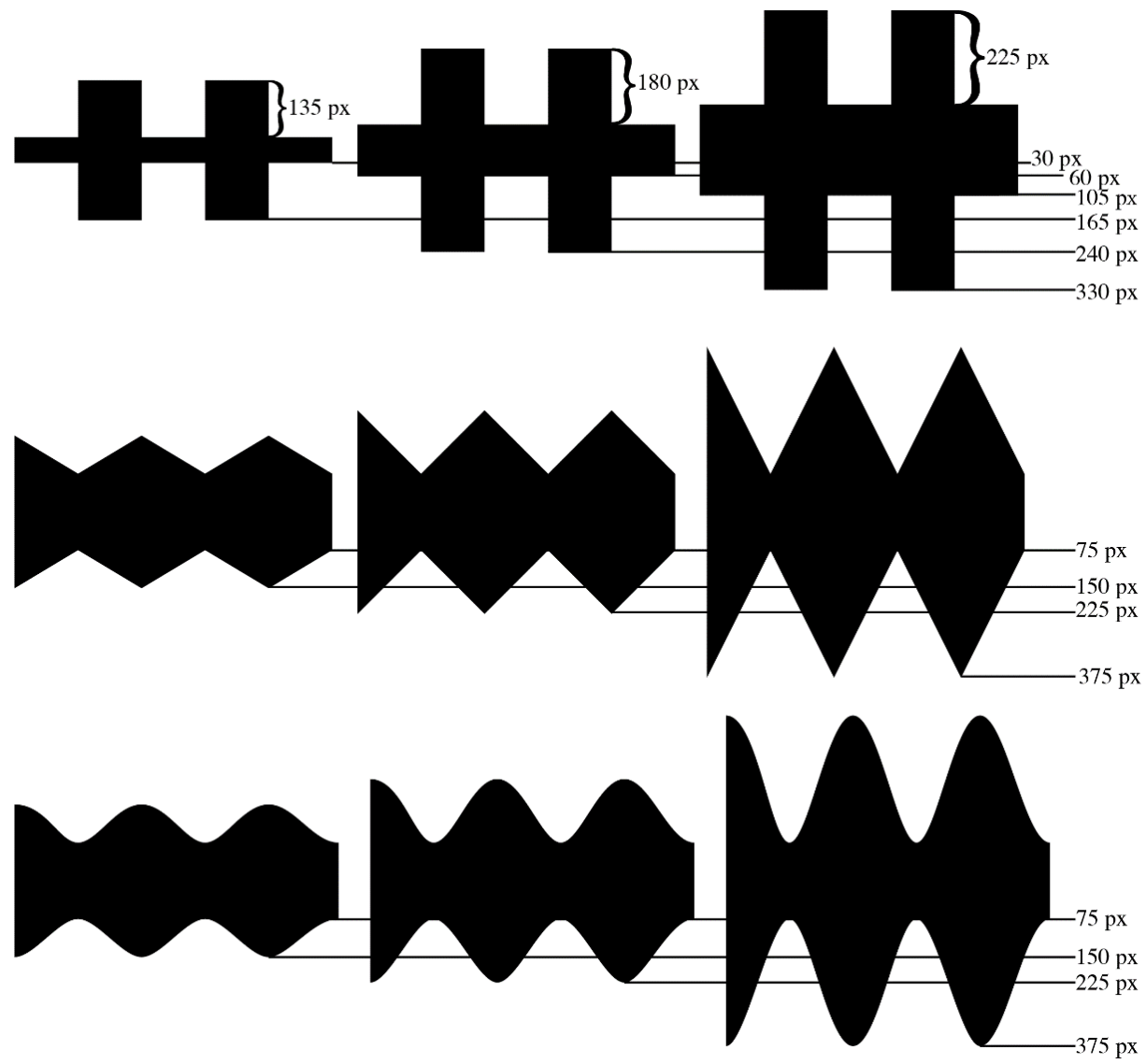

Figure 21:Test functions: triangle (top), square (middle) and sine (bottom), showing small (left), medium (middle) and large (right) sizes. The pixel values indicated are calculated from the center of the function. 


\subsubsection{Triangle}

The triangle function consists of a constant positive slope, followed by an inverse, constant negative slope. We chose this function in order to measure accuracy when the target is changing by a fixed amount over time. We were interested in participants' ability to maintain a continuous rate of change using the device. Also of interest was the ability to contrast input accuracy between situations of increasing and decreasing target size.

\subsubsection{Square}

The square function consists of two amplitudes, a minimum, and a maximum, with the target amplitude alternating between them. The square function serves two purposes: to simply observe user's ability to maintain a single amplitude of input over a given time period (static accuracy), and to observe user's ability to rapidly and accurately change between two amplitudes of input (dynamic accuracy), as a function of the size of the jump between amplitudes.

Unlike the triangle function, which maintains the same minimum amplitude, altering only the maximum, each square function has its own minimum and maximum. There is a trade-off here; ideally, all measures of dynamic accuracy would begin from the same point. However, we chose to privilege the measurement of a larger range of static accuracies over measuring dynamic accuracy. In order to get data on as large a spread of static targets as possible, we chose to measure 6 different static targets ( 2 for each function size). The size of the difference between the minimum and maximum increased with function size (Figure 21, top), allowing us to measure 3 different dynamic accuracy conditions. 


\subsubsection{Sine}

In the sine function condition, the target amplitude is described by a sine function, adjusted so that the target has the same range of values as the triangle function of the same size. The most common current use for pressure-based stylus augmentation is dynamically varying stroke width. Because varying stroke width generally does not involve the kinds of sudden changes associated with the triangle and square functions, the sine function is closer to everyday use of pressure-based stylus augmentation than the square or triangle functions.

\subsubsection{Practice Functions}

Before each set of size-based trials associated with a given function, participants were asked to complete a trial with a practice function of the same type. These practice functions have different wavelengths and amplitudes from any of the three other functions, to minimize the effect that a practice function might have in improving performance on any particular size condition.

\subsubsection{Constant Wavelength}

We chose to keep the wavelength of each function a constant 202 pixels (with the exception of the practice function, which had a wavelength of 303 pixels). In the case of the square function, the wavelength is relatively inconsequential, so long as it is large enough to allow users to stabilize their input after performing the dynamic changes between plateaus. In the case of the triangle and sine functions, the wavelength is more relevant, but we argue that in these cases, it is only necessary to change one of either the wavelength or the amplitude of the function. If we changed both the wavelength and the amplitude, we can arrive at a congruent shape, with simply a different overall size. For 
instance, if we double the amplitude of a triangle function, the slope is equivalent to a case where we halved the wavelength.

\subsection{Hypotheses and research questions}

The distance of travel of a commercial pressure-augmented stylus is very small; the hand moves less than $1 \mathrm{~mm}$ between completely depressing the pressure sensor and releasing pressure. Compared to this distance of travel, the amount of physical movement between a neutral and high-input state with the FlexStylus is significantly higher $(>1$ $\mathrm{cm})$. We hypothesize that the increased effort required to move the FlexStylus, rather than making changes more difficult, will make the device more precise $(\mathrm{H} 1)$. The source of this hypothesis is prior research on control-display gain, which indicates that as a general principle, a reduction in gain between the physical distance of input device motion results in an improvement in accuracy [15].

Prior research [55] has demonstrated that pressure input is strongly unidirectional-pressure input tends to be more accurate when participants are increasing pressure than when they are reducing pressure. We anticipate that input using the FlexStylus will exhibit a smaller unidirectional tendency than pressure input $(\mathrm{H} 2)$. Stylus pressure is an inherently asymmetrical input mechanism - when one is pressing against the screen, one has the passive haptic feedback of the screen resisting the force of the hand. This feedback does not exist when one is pulling away. We anticipate that the builtin haptic feedback of the flexible stylus in both directions will reduce asymmetry between increasing and decreasing inputs as compared to the pressure stylus. The triangle and sine target functions (described in sections 5.3.1, 5.3.3), since they represent a 
symmetrical increase and decrease in input, will allow us to analyze the directionality of the precision associated with each type of input.

We chose the square function (described in 5.3.2) to test dynamic accuracy-participants' accuracy while rapidly changing input, and static accuracy - participants' accuracy while maintaining a fixed input. We were interested in whether the magnitude of the static input had an effect on input accuracy. With respect to dynamic accuracy, we considered it likely that for both conditions participant error would increase based on the distance travelled (H3). This is informed by Wobbrock et al.'s reformulation of Fitts' law [53], which posits a positive relationship between distance travelled and error rate in pointing tasks. While our task does not match a Fitts' task exactly, it would be very surprising to find the magnitude of error not adhering to its general principles. While we anticipated a relationship between distance and degree of error, we did not hypothesize a particular specific effect. For the sake of future interaction technique designs, we were interested in determining whether or not the type of input device used had an effect on the relationship between amplitude of dynamic change and accuracy.

\subsection{Input mapping}

We transformed the raw input values from both devices, so that the maximum pressure or deformation would provide approximately equal values that were $40 \%$ larger than the maximum target value. The mappings were approximately, not exactly, equal because the flexible stylus does not have an exact "maximum" value in the way the pressure stylus does. There is no mechanism that prevents users from increasing bend beyond a threshold, so we imposed a maximum value through mapping. Note that there is a theoretical threshold to the FlexStylus, which is reached when the fibre breaks. We 
have not tested it as we have no interest at the moment in breaking our prototype. In the interests of keeping the prototype functional for the duration of the study, we increased the $\mathrm{C} / \mathrm{D}$ gain of the prototype. This kept bend input within a small range, ensuring that participants did not accidentally bend the device too far and cause a fibre to break. The Microsoft Surface Pro 2 stylus is capable of discriminating between 8 bits (1024 discrete) values of pressure input. Because of the way that we calculate input from the FlexStylus, input values are not discretized in the way that they are with the pressure stylus. However, both devices were capable of registering participant at a fine enough resolution that we did not consider the minimum input threshold to be a determining factor for participant accuracy.

\section{Trials}

As stated, the experimental design was 2 (input devices) x 3 (functions) x 3 (sizes). Each trial consisted of 6 repetitions of the same combination of function and size. We removed the first and last repetition from each trial, leaving 4 repetitions. The first repetition was removed so as to begin measurements when participants were comfortable with the trial. The last repetition was removed to reduce any effect that the approaching end of the trial might have on results. The study design resulted in 324 trials, of which 2 $(0.6 \%)$ were missing at random due to recording error. We took a measurement each time the screen updated, which occurred approximately every 50ms. Overall the experiment resulted in 260,176 data points being measured ((18 trials * 18 participants -2 trials $) *$ 808 data points per trial). 


\subsection{Participants}

18 participants took part in the study. We recruited participants through online postings, as well as through posters. The majority of participants were university students, though this was not a requirement. We recruited only right-handed participants who were physically able to grip a pen. The participants' ages ranged between 18 and 39, and the mean age was 23 (std. dev. 5.56). 10 of the participants were female. 9 participants had used a pressure-augmented stylus before, though only 2 participants used the pressure functions of a pressure stylus with regularity. 7 participants had previously participated in a study involving deformation as an input method, though none of the prior studies involved a deformable stylus. Participants were compensated $\$ 10$ for participating in the study. 


\section{Chapter: Results}

\subsection{Quantitative results}

\subsubsection{Preprocessing of Data}

We took a measurement of the user's input level each time the screen was updated, which was approximately every $50 \mathrm{~ms}$. The resulting measurements were compared against the target value to create a measure of accuracy that represented the absolute distance in pixels between the user's input and the target. We used this measurement to determine what effects the various testing factors had on accuracy.

We did not remove any outliers from the data. There were a few trials where the mean accuracy was outside of 3xIQR of the mean accuracies for all of that participant's trials, but we included these trials in our analysis because they cannot be explained by equipment or experimental error. They were simply cases where the participant had more difficulty than usual in matching the target stroke. We did not observe a particular pattern to outliers, aside from a disproportionate number (50\%) of outliers being connected to a single participant, who also had the highest mean distance from the target. The above suggests that the outliers still represented valid trials, and should be included in the data; we did not want to artificially exclude the data of participants who struggled to match the target. Outliers were relatively even between the input type conditions (Figure 22). 


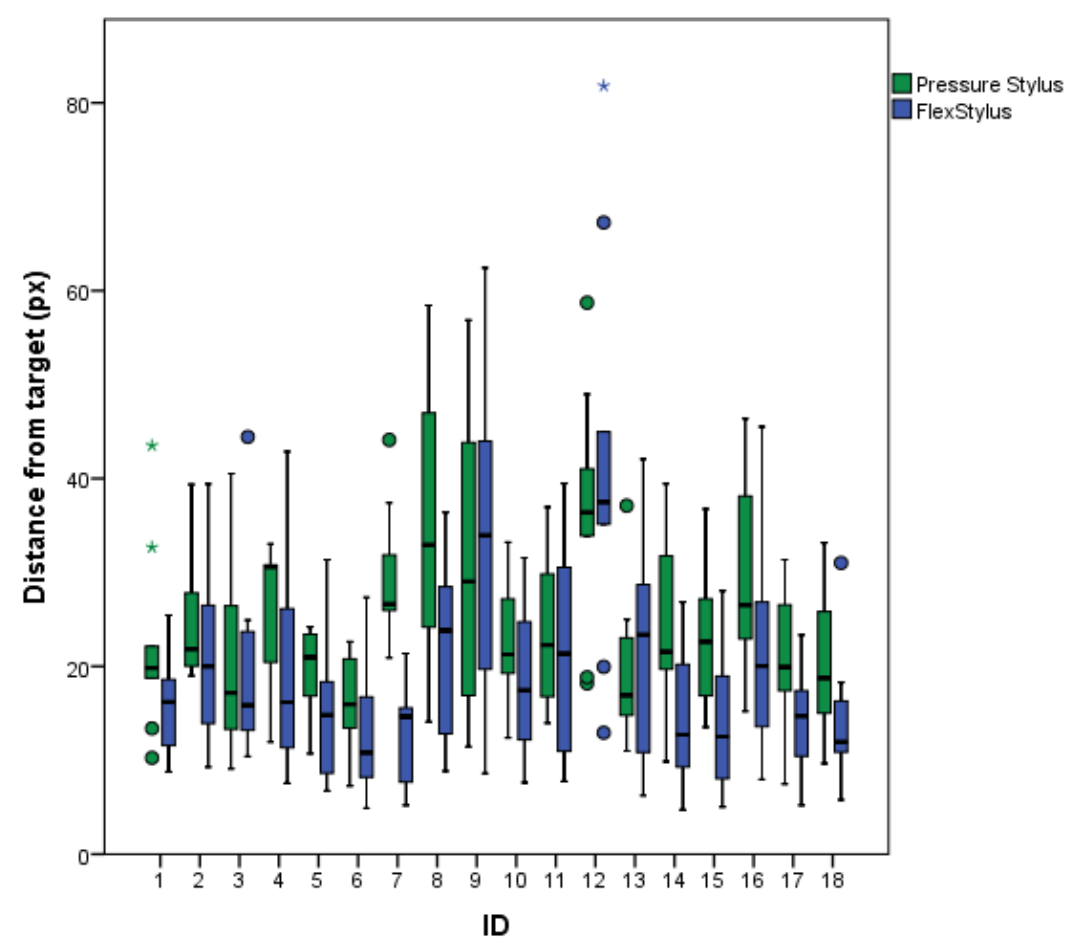

Figure 22: Boxplots of distance from target collapsed across factors, by participant

\subsubsection{General Accuracy}

We performed a repeated measures factorial ANOVA to determine the effect of the conditions (input device (2), target function (3), and function size (3)) on participant absolute accuracy (expressed as average absolute distance from target in pixels $(1 \mathrm{px}=$ $0.12 \mathrm{~mm})$ ). In order to confirm or deny our hypothesis that the FlexStylus was more accurate overall, we were interested only in studying the effect of the input device condition, not function or function size. For extra information, we chose to also look at interactions between conditions that included input device. It was likely that the other conditions would have an effect on overall accuracy, but we were interested in comparing the FlexStylus device to the pressure stylus across conditions. We found that input device had a significant effect on accuracy $(\mathrm{F}(1,15)=11.13, p=.005)$. The input device used was also associated with a large effect size (partial eta $\left.{ }^{2}=.876\right)$. None of the interactions 
between factors involving the input device condition were significant. Figure 23 displays a boxplot of mean accuracies collapsed across size, illustrating the FlexStylus condition's lower mean distance from target under all function conditions.

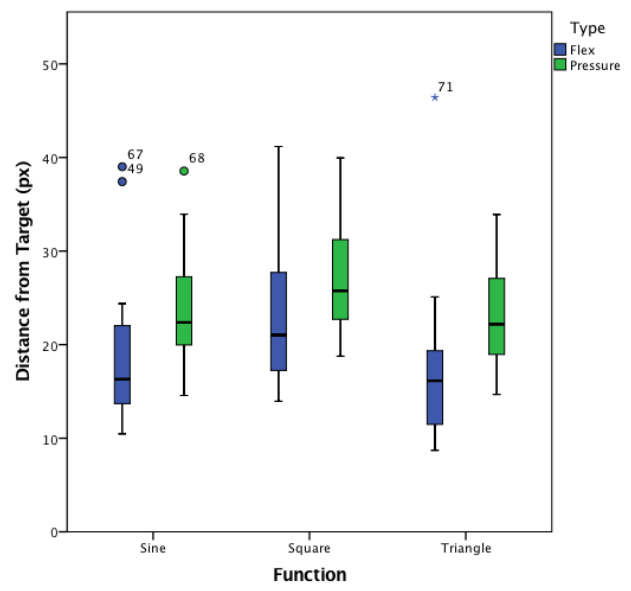

Figure 23: Boxplots of distance from target by input device and function, collapsed across size

In addition to input device, the function and size conditions had significant effects on accuracy (function: $\mathrm{F}(2,30)=10.9, p<0.001$; size: $\mathrm{F}(2,30)=86.8, p<0.001)$. However, these measures are not particularly useful in themselves for the design of interaction techniques given the lack of significance in the interaction of those factors with input device. In the next two sections (6.2 and 6.3), we isolate sections of different function conditions to examine differences in accuracy between the FlexStylus and the pressure stylus in specific contexts.

\subsection{Bidirectionality}

We were interested in determining the bidirectional accuracy of the FlexStylus compared to the pressure stylus. In other words, we wanted to discover if an increasing or decreasing target slope had an effect on accuracy, and if so, if this effect differed depending on the input device used. We performed a repeated measures factorial ANOVA with three conditions: input device (2), function (2) (we studied only the 
triangle and sine conditions for this test), and whether the target was increasing or decreasing (2). We found that directionality had a significant effect on accuracy $(F(1,17)$ $=52.580, p<.001)$, with a strong effect size $\left(\right.$ partial eta $\left.{ }^{2}=0.756\right)$. We found, predictably given earlier tests, that input device had a significant effect $(\mathrm{F}(1,17)=14.126, p=.002)$, with a still large, but smaller effect size than slope (partial eta $\left.{ }^{2}=0.454\right)$. However, there was no statistically significant interaction between the input device used and whether the slope was positive or negative. This suggests that both means of input are similarly nonbidirectional. Examining a plot of the data (Figure 24) provides evidence for this: While the pressure stylus has a larger overall distance from the target, the effect of directionality on accuracy is similar for both input devices.

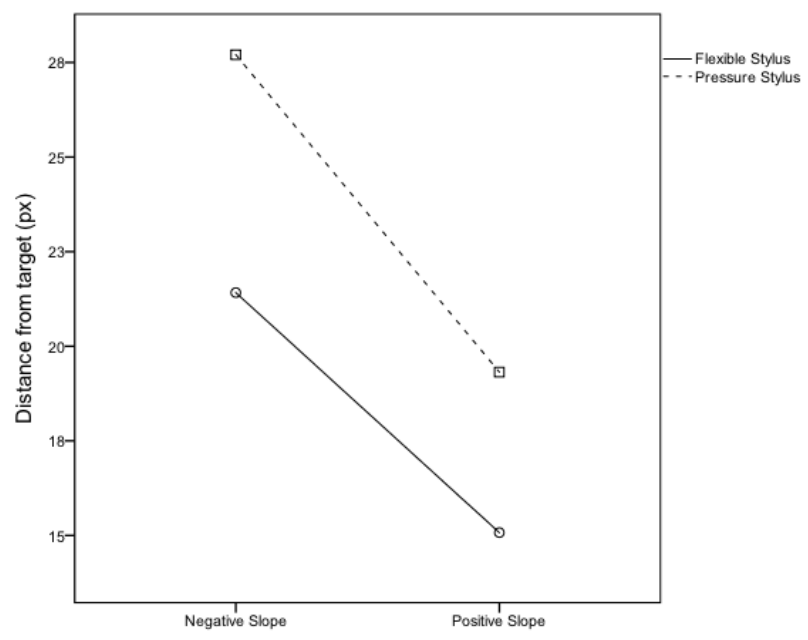

Figure 24: Estimated Marginal Means of distance from target by slope (sine and triangle functions)

\subsection{Square wave condition: static and dynamic accuracy}

We designed the square wave condition to test dynamic and static accuracy. While we found that input device had a significant effect on both dynamic and static accuracy, this was not our interest, having already determined that input device had a strong effect on accuracy in general. 


\subsubsection{Static Accuracy}

We used 6 different static target amplitudes with the square wave condition in order to determine if there was any correlation between static accuracy (accuracy when the target is not changing) and target amplitude, and whether that correlation was different between the two devices. In other words, we wanted to determine if the amplitude of a static target had any effect on participants' ability to match that target, and if this effect was altered based on input device. We pre-processed the collected square wave trials to remove a buffer area of 20 pixels in each direction around each change in target size. We removed this area from analysis in order to reduce the effect of correction after rapid changes in input. The blue area in Figure 25 represents the area used to study the static accuracy. We performed a repeated-measures factorial ANOVA with two factors. The two factors were the input device used (2), and the static target size (6), consisting of the 6 categories of possible static targets (see Square Wave, section 5.3.2 and Figure 21). Neither the size of the target nor the interaction between input device type and target size had a statistically significant effect on input accuracy. The interaction between input device type and target size was close to significance $(p=.07)$, which suggests that there may be an interaction between input device and target size, but our test was unable to capture it.

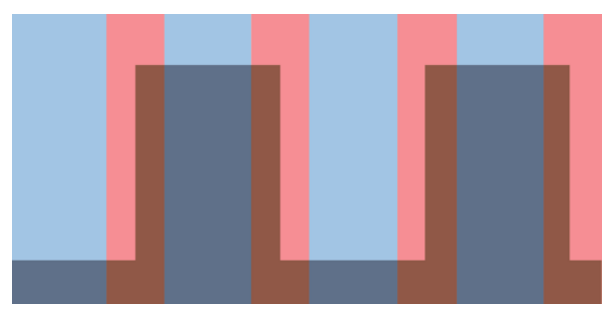

Figure 25: Square function, showing areas studied for static (blue) and dynamic (red) accuracy 


\subsubsection{Dynamic Accuracy}

We had anticipated that there would be a connection between distance travelled and dynamic accuracy (H3). We were interested in determining whether the type of input device would have an effect on the relationship between distance of travel and input accuracy. We used the separated out dynamic segments (Figure 25, red) - in which the target rapidly shifts by a specified distance - from the square function condition (using the 20 pixels excluded from the static accuracy test), and ran a repeated-measures factorial ANOVA with two factors: input device (2), and distance (3: $135 \mathrm{px}, 180 \mathrm{px}, 225$ $\mathrm{px})$. Distance was, as predicted, a strong determinant of input accuracy $(\mathrm{F}(1.62$, $34)=31.17, p<0.001$, partial eta $\left.^{2}=.647\right)$. We also found a statistically significant linear correlation between distance condition and input accuracy $(\mathrm{F}(1)=63.061, p<.001$, partial $\mathrm{eta}^{2}=.788$ ), indicating that as distance increases, accuracy decreases, confirming $\mathrm{H} 3$. However, we did not find a significant interaction between input device and distancedistance had a similar effect on accuracy regardless of which stylus was used.

\subsection{Image Overlays}

In order to get an overview of all participants' performance during a given set of conditions, we created overlays for each trial, consisting of all of the participants' input data for a given trial superimposed on one another (Figure 26). We used a multiply blending function in order to make the differences between the participants' trials more prominent. Examining these images, trends emerge that support our quantitative analysis. Based on the relative similarity of the resulting images to the target functions, it visually appears that the flexible stylus is more accurate across all conditions. Examining the square condition for both input devices, we can see that participants tended to overcorrect 
on dynamic input-On the leading edge of the square waves, the participants tended to provide too much input, and as they reduced the input, they tended to provide too little. This creates the "spikes" that precede, and the "gaps" that follow, each plateau. It also appears that in the pressure condition, participants had greater difficulty with stabilizing their input after returning to the lower target level.

\section{Pressure:}
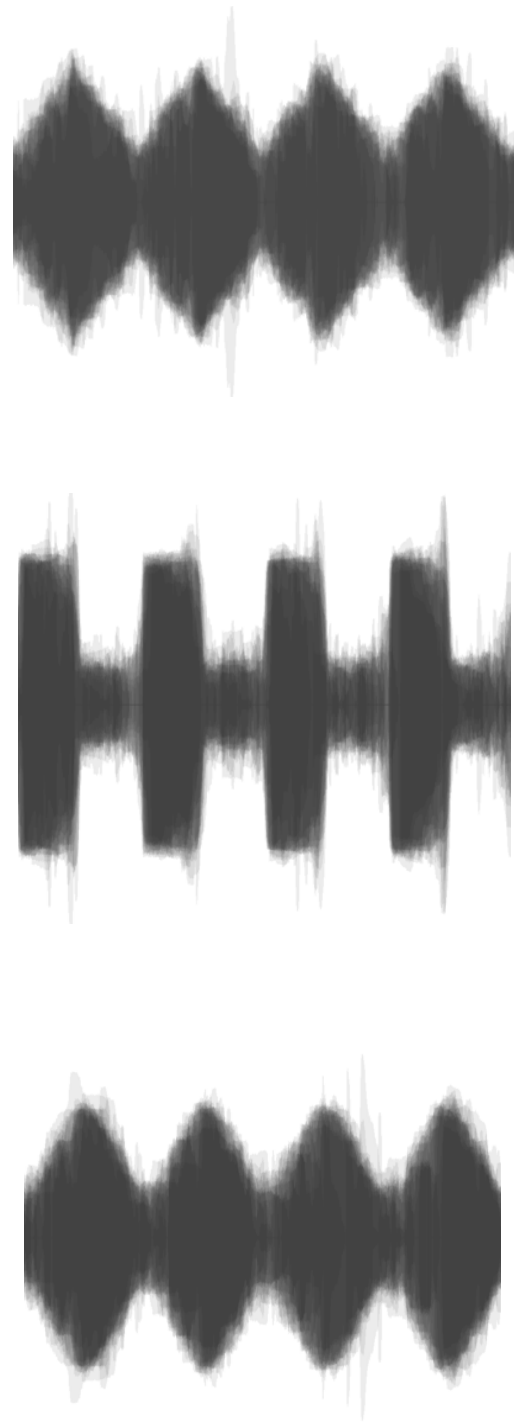

Flex:
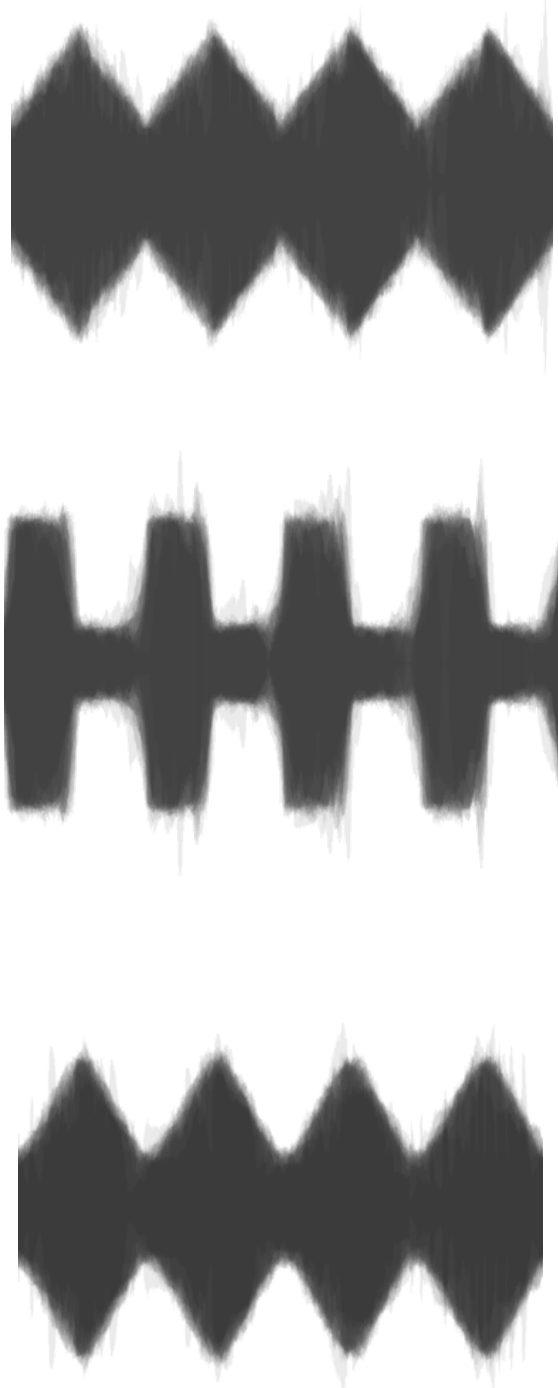

Figure 26: Superimposed trial images, pressure stylus (left) and FlexStylus (right), medium size condition, for the triangle (top), square (middle), and sine (bottom) functions 


\subsubsection{Stepped effect with pressure stylus}

Participants using the pressure stylus frequently exhibited a "stepped" effect (Figure 27) during the sine and triangle conditions. Rather than smoothly adjusting the amount of pressure to match the target, their input consisted of a series of discrete steps to increase or decrease the pressure, with short plateaus of steady pressure in between. We examined images of all trials with the sine and triangle function conditions (216 images). None of the trials with the FlexStylus had showed a stepped shape-when participants were inaccurate with the FlexStylus, it presented in spikes rather than plateaus. Of the 108 pressure trials, 66 of them (61\%) demonstrated, to some extent, a stepped effect.

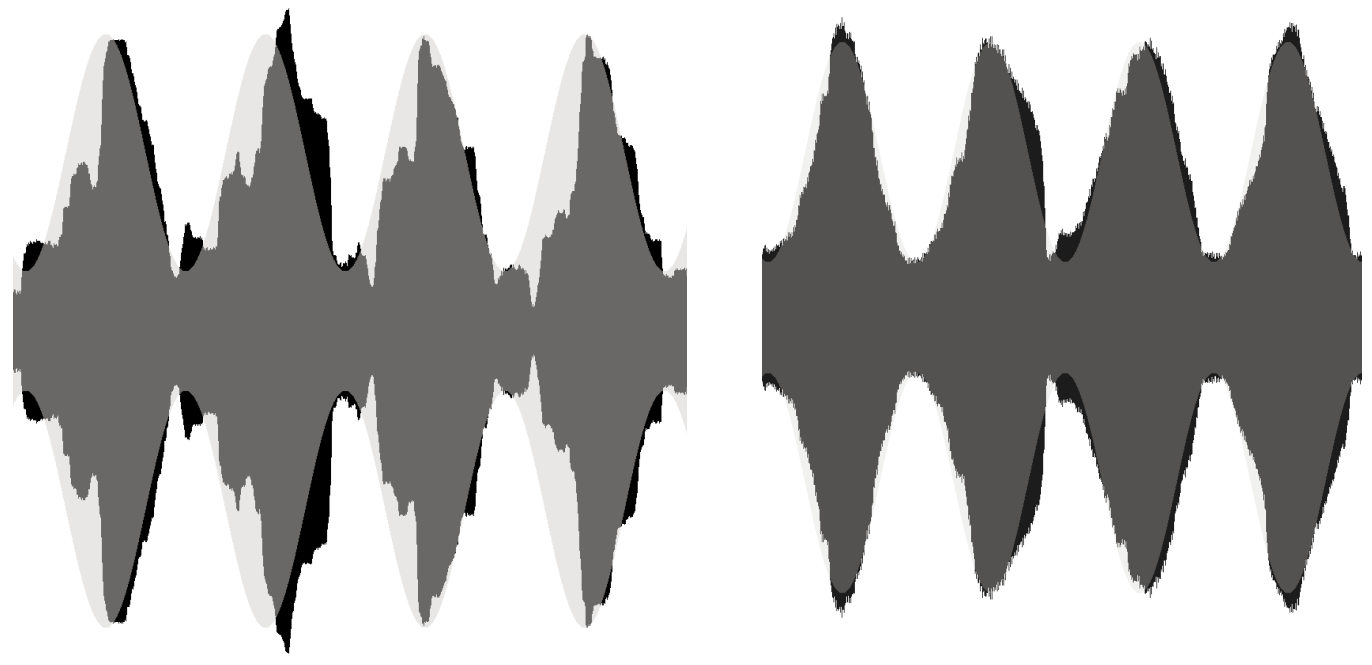

Figure 27: Trial data for one typical participant demonstrating stepped pattern with the pressure stylus (left). The same participant is not demonstrating the pattern when using the FlexStylus (right).

\subsection{Perceptual results}

\subsubsection{User Preference}

We asked participants to indicate which device that they preferred according to three criteria: accuracy, comfort, and overall preference. Each question had three potential responses: preference for the FlexStylus, preference for the pressure stylus, and 
no preference (Table 1). Removing the no-preference responses and performing a binomial test of proportions, we found a statistically significant preference for the FlexStylus in the categories of accuracy $(p<.001)$ and overall preference $(p<.021)$. User preference in the category of comfort was not found to have significance.

Table 1: Counts and percentages of participant device preference

\begin{tabular}{lllll}
\hline Criteria & FlexStylus & Pressure Stylus & No preference & Binomial sig. \\
\hline \hline Accuracy & $17(94 \%)$ & $1(5 \%)$ & - & .000 \\
Comfort & $10(55 \%)$ & $7(39 \%)$ & $1(5 \%)$ & .629 \\
Overall & $13(72 \%)$ & $3(17 \%)$ & $2(11 \%)$ & .021 \\
\hline
\end{tabular}

Participants were generally accurate in their self-assessment of relative accuracy. The one participant who assessed their accuracy with the FlexStylus as lower than the pressure stylus did have a lower overall mean actual accuracy using the FlexStylus. Two participants misjudged their accuracy with the FlexStylus as higher than with the pressure stylus (their actual accuracy was higher with the pressure stylus, but they perceived it otherwise).

\subsubsection{Prototype Properties}

We asked participants three questions relating to the FlexStylus prototype. The questions each had three possible responses: "I would prefer the device to be thicker, thinner or neither," "I would prefer the device to be longer, shorter, or neither," "I would prefer the device to be more flexible, less flexible, or neither." Participants responded predominantly with "neither" for all three questions (see Table 2). 
Table 2: Participant Responses regarding physical properties of FlexStylus prototype

\begin{tabular}{llll}
\hline Thickness: & Thinner & Neither & Thicker \\
& $2(11 \%)$ & $15(83 \%)$ & $1(5 \%)$ \\
\hline Length: & $\underline{\text { Shorter }}$ & $\underline{\text { Neither }}$ & $\underline{\text { Longer }}$ \\
& $4(22 \%)$ & $12(67 \%)$ & $2(11 \%)$ \\
\hline Flexibility: & Less Flexible & Neither & More flexible \\
& $4(22 \%)$ & $9(50 \%)$ & $5(28 \%)$ \\
& & & \\
\hline
\end{tabular}

\subsubsection{Likert-Style Responses}

\begin{tabular}{ll} 
Feedback matched input: & Pressure: \\
& Flex: \\
\hline Difficult to change stroke width accurately: & Pressure: \\
& Flex: \\
\hline Device was easy to learn how to use: & Pressure: \\
& Flex: \\
\hline Would use a similar device, if available: & Pressure: \\
& Flex: \\
\hline Device was physically difficult to use: & Pressure: \\
& Flex: \\
\hline Device was enjoyable to use: & Pressure: \\
& Flex: \\
& Strongly Disagree \\
& Disagree
\end{tabular}

Figure 28: Graph of Likert Responses

Participants had a general overall preference for the FlexStylus in response to the following questions: "I found the stroke (grey area) clearly matched the movements of my hand," and "I found the device to be enjoyable to use," as illustrated in Figure 28. With respect to the FlexStylus, participants also tended to disagree more with the statement that it was difficult to change the magnitude of input. However, they found the FlexStylus slightly more physically difficult to use, and they also had a larger tendency to 
disagree with the statement that they would use a similar device if possible. We propose that both of these results are connected to the fact that the FlexStylus is a prototype. This will be discussed in chapter 7 . 


\section{Chapter: Discussion}

The results of this study, both in participant data and qualitative responses, strongly suggest that deformation is a worthwhile means for providing parametric input in a drawing application to change stroke width. More generally, absolute deformation appears to be a promising interaction technique for providing accurate input in a tablet context, which could be exploited for interactions beyond parametric input, such as menu navigation.

We can use the results of the study to inform future interaction techniques, and to confirm the utility of interaction techniques already developed. For example, we suggested that a tool grip menu item could use continuous analog deformation input to control a slider selecting brush size (see 4.2.2). Results suggest that the deformation stylus would enable users to make these kinds of selections with more precision than a comparable menu selection based on pressure.

\subsection{Improved Performance with Flexible Stylus}

We confirmed our hypothesis (H1) stating that participants would exhibit improved performance with the FlexStylus. We offer two explanations for improved participant performance with the FlexStylus as compared to the pressure stylus. The first is $\mathrm{C} / \mathrm{D}$ gain - the fact there is a larger motion in real space associated with the FlexStylus than with the pressure stylus. The second explanation is the continuous haptic feedback that the FlexStylus provides to the user.

As mentioned previously, the C/D gain of the FlexStylus was set higher than necessary in order to ensure that participants would not over-bend the device and damage the sensors. Despite this, the FlexStylus has a much smaller C/D gain than the pressure 
stylus, since - while the pressure stylus does translate linear motion into input - the actual distance of this motion is almost imperceptible to participants. Since a larger movement in space translated to the same amount of input on the screen, it was easier for participants to distinguish between levels of flex input, as opposed to pressure. We think it is possible that choosing a less conservative C/D gain may result in further improvements to accuracy, but the optimum mapping still must be studied.

Another explanation for the improved performance with the FlexStylus is the inherent haptic feedback of the device. One participant verbally alluded to this inherent haptic feedback during the conducting of the study. The participant stated that it was easier to remember how much to bend the device as opposed to how much pressure to apply, because they could remember the position of their thumb in space, rather than having to rely on the visual feedback to determine input magnitude. This supports previous research [1], as well as general theory behind the choice of deformation as an interaction method, which states that the inherent (passive) haptic feedback improves users' ability to bend devices to a specific magnitude. We believe that the fact that the physical configuration of the device changed as input changed allowed the participants of the study to have a better feeling of how much input they were providing with the FlexStylus.

Our second hypothesis $(\mathrm{H} 2)$, that the Flexstylus would exhibit a smaller variation in accuracy than the pressure stylus depending on if the target slope was increasing or decreasing, was not supported. We suggest this effect is because while the user is releasing bend input, the fingers and thumb do not experience resistance from the FlexStylus. The resulting lack of feedback is parallel with the pressure stylus. In the case 
of the pressure stylus, the hand holding the device does not experience the resistance of the tablet screen while releasing pressure. We thought the directional effect would be reduced by the user's ability to feel the stylus's bend configuration in the hand at all times, but our data suggests this is not the case. That the FlexStylus' input accuracy is affected by positive or negative direction is important information for designing interactions on the FlexStylus, as it will be necessary to avoid interactions in which the user is forced to reduce the amplitude of bend input in a precise way. All of our proposed menu-related interaction techniques (see Chapter 4) involve making selections by bending the stylus away from a neutral bend state, so increases in bend are much more likely than decreases. We suggest that future interactions be designed to continue this pattern.

\subsection{Properties of Pressure Input}

While less relevant to the FlexStylus prototype, our results support two properties of pressure input previously mentioned in the literature. The first is that accuracy using pressure input is not equally bidirectional [55], with significantly reduced performance when the target slope was negative. The other is that participants can distinguish between a limited number of levels of pressure [33]. While examining recorded participant trials, we remarked that a number of them exhibited a similar staircase (stepped) effect. Participants were unable to modify pressure by a smoothly increasing amount, so when performing the triangle and sine tasks, there was a continuous correction effect. Participants would maintain one level of input for a short period while target value changed, then overcorrect, allowing the target to catch up to their new level of input. Rather than a slope, participants' input was divided into a series of steps. This also 
reflects past research on pressure input, which suggests that participants are only able to distinguish between 6 levels of pressure input [55]. We consider the fact that our results have matched prior research on pressure augmented styluses to be encouraging, and it gives us confidence in those results of the study that to pertain to the FlexStylus.

\subsection{Discussion of Qualitative Results}

Participants tended to find themselves more accurate using the FlexStylus, and tended to prefer it overall. We suggest that the overall preference is strongly connected to accuracy - the study was largely confined to measuring accuracy, so participants preferred the device which they felt made them better at the study task. Further study will be necessary to determine if this reported user preference is equally applicable to other kinds of tasks, such as manipulating parameters using the device while drawing.

Participants digressed from the generally-held trend of preference for the flexible stylus in their reactions to the statement "I would use a similar device if it were available to me." Despite that a significant majority of participants had described the flexible stylus as more accurate, and preferable overall, the general opinion among participants was that they would be more likely to use the pressure-based stylus if it were made available to them. Our hypothesis for explaining this anomaly pertains to the fact that participants were evaluating a prototype device against a commercially produced device. It is possible that the question was too vague, and participants imagined a "similar device" to have all the negative characteristics associated with a prototype (e.g., larger weight, not wireless, lower-quality capacitive tip). However, this explanation is only a hypothesis, and is not definitive. It will be necessary to perform more studies in order to determine exactly 
whether this response comes from a reaction to the physical prototype or to bend itself as an input method.

We found that participants were generally satisfied with the length, thickness and flexibility of the device. We believe this is because in the prototype design process we attempted to emulate as closely as we could the dimensions of a commercial stylus device. Future prototypes will be designed to have similar dimensions as the FlexStylus device used for testing. 


\section{Chapter: Conclusion}

\subsection{Summary of Contributions}

This thesis presented three contributions to the domains of deformable user interface research and augmented styluses. These contributions are: the FlexStylus itself, and the associated fibre-optic technique for sensing vector deformation; a series of interaction techniques using the FlexStylus; and the study comparing input accuracy between the FlexStylus and a commercial pressure stylus.

We created a deformable stylus device augmented with four fibre-optic bend sensors, enabling two degrees of input freedom (the angle relative to the body of the device, and the absolute deformation). This detection technique improves over limitations of widely used flex sensing techniques, in that it does not limit the axis of deformation. As a result, it enables the construction of flexible devices that are less limited to specific form factors. It also allows us to prototype new interaction techniques that exploit the two degrees of freedom, for example by creating a virtual pointer for gesture and 2D parametric input, or by creating 360 degree marking menus.

We discussed 8 interaction techniques using the FlexStylus. Because the type and amount of bend input is constrained by the way that device is held, we use this gripdependency to inform our interaction techniques. We propose 3 grips, the tool grip, menu grip, and scroll grip, and demonstrate how the degrees of freedom associated with each grip can be exploited for interaction techniques. We also propose methods for inferring grip. The system can infer the scroll grip when bend input is concurrent with an absence of $x-y$ touch information. The system can infer the menu grip using a gesture that - 
owing to the different degrees of freedom available to different grips - can only be performed while holding the device in the menu grip.

We compared the FlexStylus against a commercially-available pressure augmented stylus in a task that imitated using augmented stylus input to control the width of a stroke. We found that, across conditions, the FlexStylus was associated with a lower degree of input error. The results of the study demonstrated that deformation is a promising mode for providing absolute parametric input using a stylus. However, in order to determine exactly to what extent this is true, and to explore the other types of augmentation possible using the flexible stylus, future study will be necessary.

\subsection{Limitations}

The FlexStylus prototype has numerous limitations. The most important of these is that it is corded. The extra weight of the cord on the base of the device is especially problematic because, depending on the user's grip, this weight can influence the bend state of the device. It was necessary, during the study and while testing interactions, to use a stand to hold the cord above the device, thus preventing its weight from affecting input. A second limitation is the tip of the device. For simplicity, and to allow us to test the device on any capacitive-sensing touchscreen, we used a conventional aftermarket capacitive tip. However, this type of stylus tip, compared to an active sensing tip used in higher end stylus devices, offers limited precision and creates problems with palm rejection. The prototype was also limited in that it was delicate; concerns about breaking the prototype caused us to increase the C/D gain of the FlexStylus in order to ensure that participants would not flex the device to an extent that might damage the prototype. It is 
possible that this limitation prevented us from fully capturing the improvement in accuracy associated with the FlexStylus.

Our interaction techniques were not implemented within a fully functional drawing application. Because of this, we were unable to determine how effective our proposed system of differentiating between the tool and menu grip based on a flex gesture (section 4.4) actually is for a digital artist at work. We also only studied a limited number of interactions. For example, we did not implement multi-level marking menus.

The biggest limitation of the study was that, in order to have a baseline assessment of the performance of the device, we omitted certain factors, such as providing input to the device while simultaneously changing $x-y$ position. Therefore, the study did not perfectly mimic an actual use-case scenario of a digital art device. The study also did not evaluate the FlexStylus' secondary degree of freedom - angular detection of deformation.

\subsection{Future Work}

A qualitative result that merits future work is the difference between participants' responses describing FlexStylus as being more accurate and preferable overall to the pressure stylus, and their responses to the question of whether or not they would use a similar device if it were available to them. It will take an improved prototype and further study to determine whether this response was simply an artefact of the particular prototype itself, or whether there is some intrinsic aspect to deformation input using a stylus that causes participants to be less interested in incorporating the device into their lives. 
With respect to interaction techniques, future work involves implementing multilevel marking menus, allowing for flex-gesture based input without visible menus for expert users. We are interested in determining if the benefits of these menus for mouse devices apply equally to interactions with the flex cursor. We would also like to implement scroll grip interactions beyond simple scrolling, such as rotating an object in 3D space, or temporally scrolling through animation frames.

Future study will examine parametric input occurring simultaneously to changes in the $x-y$ position of the device, to more closely match situations where a user is modifying brush parameters while drawing. We are also interested in evaluating users' accuracy using the angular deformation degree of freedom. In order to perform a study comparing the FlexStylus to previously studied styluses, we will compare angular deformation input against a suitable pre-existing augmented stylus input type. We propose a tilt detecting stylus, owing to the fact that a tilt-detection is another type of stylus augmentation that offers directional control.

Aside from additional studies, future work will involve incremental improvements to the prototype device. One area of improvement is robustness. The aspect of the prototype which could be most improved for robustness is the flexible housing, which in its current form uses a piece of rubber tubing. Its robustness can be improved by using a molded silicone part. Another area of improvement for the prototype is to alter the method of $x-y$ detection. It currently uses a capacitive tip, which is less precise than a more sophisticated active or magnetic solution. The prototype might also be improved by better managing the cabling associated with it—an ideal FlexStylus would be wireless. In the interaction technique for selecting from a range of $2 \mathrm{D}$ continuous values (4.3.2), we 
noted that the button we had affixed to the FlexStylus was not ideal, because depressing the button slightly altered the device's bend state. Future iterations will use a capacitive touch pad rather than a button.

Finally, and most importantly, we would like to develop the FlexStylus and associated software to the point it can be studied in its intended context-digital art. We would like to be able to allow digital artists to perform their work with the FlexStylus and a system incorporating the interaction techniques described in this thesis. This will allow us to more fully evaluate its effectiveness as a tool for enhancing the experience of digital art and facilitating creative expression. 


\section{Appendices}

\section{Appendix A Study Materials}

\section{A.1 Participant Questionnaire}

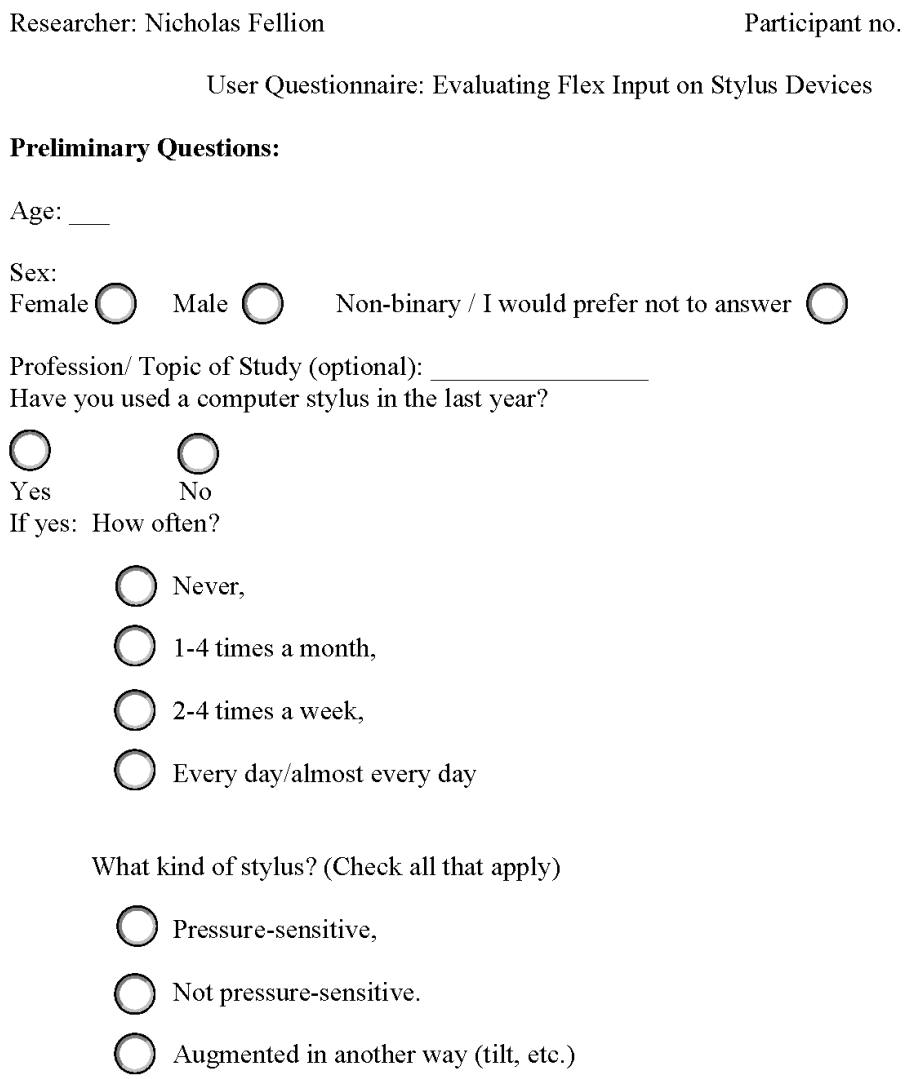

If you know the brand/model of your tablet/stylus, please provide below:

Have you used hardware before (in a study or otherwise) which made use of bend gestures?

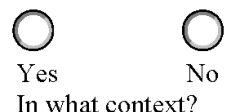

In what context? 


\section{A.2 Pressure stylus Likert-style survey}

\section{Pressure-based stylus:}

I found the device to be enjoyable to use:

It was physically difficult to use the device:

I would be interested in making use of a similar device if it were available:

$\bigcirc_{\text {Strongly Disagree }} \bigcirc_{\text {Disagree }} \underset{\text { Agree }}{O}$

I found the device difficult to learn:

$\bigcirc_{\text {Strongly Disagree }} \bigcirc \underset{\text { Nisagree }}{0}$

I found it difficult to accurately change the degree of pressure (stroke width):

Ifelt the stroke (grey area) matched closely the movements of my hand:




\section{A.3 FlexStlyus Likert-style survey}

\section{Flexible Stylus:}

I found the device to be enjoyable to use:

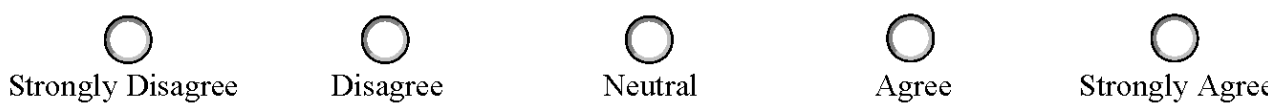

I would be interested in making use of a similar device if it were available:

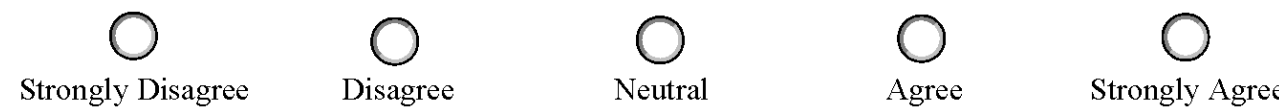

It was physically difficult to use the device:

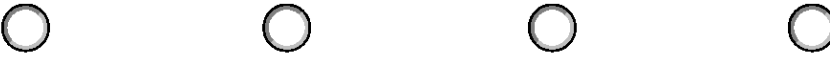

Strongly Disagree

Disagree
Neutral

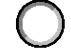

Neutral

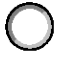

Agree

\section{Strongly Agree}

I found it difficult to accurately change the degree of bend (stroke width):

Ifelt the stroke (grey area) matched closely the movements of my hand:




\section{A.4 FlexStylus Prototype Feedback}

Physical Properties of the device:

I would have preferred the stylus prototype to be:

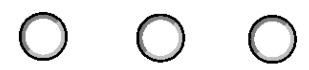

Thicker, Thinner, Neither

Specify: (optional)

I would have preferred the stylus prototype to be:

Longer, Shorter, Neither

Specify: (optional)

I would have preferred the stylus prototype to be:

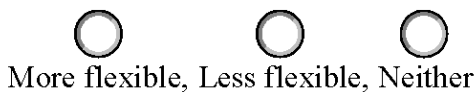

More flexible, Less flexible, Neither

Specify: (optional)

\section{Open-ended questions:}

Are there specific aspects of the flexible stylus which contributed to your enjoyment using it?

Are there specific aspects of the flexible stylus which took away from your enjoyment using it?

What applications can you imagine the flexible stylus to be well-suited for? 


\section{A.5 Comparison Questions}

\section{Comparison Questions:}

Which device did you think allowed you to match the target more accurately?

Flexible Stylus

$\bigcirc$ Pressure-Based Stylus

Neither

If you have any comments regarding why this might be, please put them here:

Which device did you find to be more comfortable to use?

$\bigcirc$ Flexible Stylus

$\bigcirc$ Pressure-Based Stylus

Neither

If you have any comments regarding why this might be, please put them here:

Which device did you prefer overall?

$\bigcirc$ Flexible Stylus

$\bigcirc$ Pressure-Based Stylus

No Preference

If you have any comments regarding why this might be, please put them here:

Please place any general comments you may have regarding the study here: 


\section{A.6 Consent Form}

Carleton

Canada's Capital University
Title: Evaluating Flex Input on Stylus devices

Date of ethics clearance: February $29^{\text {th }}, 2016$

Ethics Clearance for the Collection of Data Expires: December 31 $1^{\text {st }}, 2016$ , choose to participate in a study on flex gestures on computer styluses. This study aims to determine user performance when performing flexion gestures on a computer stylus device. The researcher for this study is Nicholas Fellion in the Carleton University School of Information Technology department.

$\mathrm{He}$ is working under the supervision of Audrey Girouard in the School of Information Technology, and this research is funded by an NSERC Discovery Grant.

This study involves one 60 minute session. With your consent, your hands will be video recorded. Also, with your consent, images will be taken which will may be used to illustrate the study. No images of the participants will be distributed other than of the hands, and these images will be converted to basic line drawings that will not contain any identifiable features. There are no known risks to taking part in this study.

The following describes in detail the study procedure:

Participants will be invited to sit at a desk holding a tablet computer and a flexible stylus device. Participants will be reminded by researcher that participation is completely voluntary and can be ended at any time. Participants will then be asked to sign consent forms.

The study will consist of an image of a pen stroke displayed on the screen of the tablet, which participants will be asked to replicate using the flexible stylus prototype. There will be various stroke parameters which will be modified by the manipulation of the stylus. The stylus can be moved in the $x-y$ axes on the screen, as well as flexed using the hand.

The data collected will consist of recorded participants' performance data, with various metrics that relate to how accurately the participants are able to replicate the stroke on the screen, as well as the time taken to replicate the stroke. Some pictures will also be taken, with participants' consent. They will be digitally altered into unidentifiable line drawings for illustrative purposes in the paper. Video may be used, but only descriptions of video will be included in study results.

As a token of appreciation, you will receive $\$ 10$. This is yours to keep, even if you withdraw from the study.

All research data, including video-recordings and any notes will be encrypted. Any hard copies of data (including any handwritten notes or USB keys) will be kept in a locked

This document has been printed on both sides of a single sheet of paper. Please retain a copy of this document for your records. 
cabinet at Carleton University. Research data will only be accessible by the researcher and the research supervisor. Research data will be anonymized by participant number. You may take breaks at any time if you experience hand stress. You also have the opportunity to withdraw from the study up until the end of the testing session.

If you would like a copy of the finished research project, you are invited to contact the researcher to request an electronic copy which will be provided to you. The data will be used for Nicholas Fellion's master's thesis research, and also may be submitted for publication in HCI conference papers.

The ethics protocol has been approved by the Carleton University Research Ethics Board- B Protocol \#15-245, on February $29^{\text {th }}, 2016$ Should you have questions or concerns related to your involvement in this research, please contact:

\section{REB contact information:}

Dr. Shelley Brown, Chair

Research Ethics Board

Carleton University

511 Tory

1125 Colonel By Drive

Ottawa, ON K1S 5B6

Tel: $613-520-2517$

ethics@carleton.ca

Researcher contact information:

Nicholas Fellion

School of Information Technology

Carleton University

nicholas.fellion@carleton.ca

Signature of participant

Signature of researcher
Supervisor contact information: Audrey Girouard School of Information Technology Carleton University 520-2600 ext. 8817

Audrey.Girouard@carleton.ca

Date

Date

I consent to video being recorded of my hands for future analysis. This is optional and separate from consenting to participation in the study.

Signature of participant 


\section{Bibliography}

1. Teemu T Ahmaniemi, Johan Kildal, and Merja Haveri. 2014. What is a device bend gesture really good for? Proceedings of the SIGCHI conference on Human Factors in computing systems: $3503-3512$.

2. Apple. Apple Pencil. Retrieved from http://www.apple.com/ca/apple-pencil

3. Arduino. Uno. Retrieved from https://www.arduino.cc/en/Main/ArduinoBoardUno

4. Ravin Balakrishnan, George Fitzmaurice, Gordon Kurtenbach, and Karan Singh. 1999. Exploring Interactive Curve and Surface Manipulation Using a Bend and Twist Sensitive Input Strip. Proceedings I3D symposium on interactive $3 D$ graphics, 111-118.

5. Xiaojun Bi, Tomer Moscovich, Gonzalo Ramos, Ravin Balakrishnan, and Ken Hinckley. 2008. An Exploration of Pen Rolling for Pen-based Interaction. Proceedings of the 21st Annual ACM Symposium on User Interface Software and Technology: 191-200.

6. Eric A Bier, Maureen C Stone, Ken Pier, et al. 1994. Toolglass and Magic Lenses: The See-through Interface. ACM, 445-446. http://doi.org/10.1145/259963.260447

7. Jesse Burstyn, Juan Pablo Carrascal, and Roel Vertegaal. 2016. Fitts' Law and the Effects of Input Mapping and Stiffness on Flexible Display Interactions. Proceedings of the 2016 CHI Conference on Human Factors in Computing Systems: 3649-3658.

8. Géry Casiez, Daniel Vogel, Ravin Balakrishnan, and Andy Cockburn. 2008. The Impact of Control-Display Gain on User Performance in Pointing Tasks. Human-Computer Interaction 23, 3: 215-250.

9. Ian Chilvers. Camera Obscura.

10. J C F de Winter and S de Groot. 2012. The effects of control-display gain on performance of race car drivers in an isometric braking task. J. Sports Sci. 30, 16: 1747-1756.

11. Alexander Keith Eady and Audrey Girouard. 2015. Caret Manipulation Using Deformable Input in Mobile Devices. Proceedings of TEI Conference on Tangible and Embodied Interaction: 587-591.

12. Matthew Ernst and Audrey Girouard. 2016. Exploring Haptics for Learning Bend Gestures for the Blind. Proceedings of the 2016 CHI Conference Extended Abstracts on Human Factors in Computing Systems: 2097-2104.

13. Matthew Ernst and Audrey Girouard. 2016. Exploring Haptics for Learning Bend Gestures for the Blind. ACM, 2097-2104.

14. Yuqiang Fan, George Wu, Wanting Wei, Yufeng Yuan, Feng Lin, and X Wu. 2012. Fiber-optic bend sensor using LP21 mode operation. Opt. Express 20, 24: 26127-26134.

15. C B Gibbs. 1962. Controller Design: Interactions Of Controlling Limbs, Time-lags And Gains In Positional And Velocity Systems. Ergonomics 5, 2: 385-402.

16. Audrey Girouard, Jessica Lo, Riyadh, Farshad Daliri, Alexander Keith Eady, and Jerome Pasquero. 2015. One-Handed Bend Interactions with Deformable Smartphones. Proceedings of the 33rd Annual ACM Conference on Human Factors in Computing Systems: 1509-1518. 
17. Ravindra S Goonetilleke, Errol R Hoffmann, and Ameersing Luximon. 2009. Effects of pen design on drawing and writing performance. Applied Ergonomics 40, 2: 292-301.

18. E Gray. 1888. Telautograph.

19. Khalad Hasan, Xing-Dong Yang, Andrea Bunt, and Pourang Irani. 2012. A-coord Input: Coordinating Auxiliary Input Streams for Augmenting Contextual Penbased Interactions. Proceedings of SIGCHI conference on Human Factors in computing systems: 805-814.

20. Gero Herkenrath, Thorsten Karrer, and Jan Borchers. 2008. Twend: Twisting and Bending As New Interaction Gesture in Mobile Devices. In Extended abstacts of CHI International conference on human factors in computing systems: 3819-3824.

21. Christopher F Herot and Guy Weinzapfel. 1978. One-point Touch Input of Vector Information for Computer Displays. ACM SIGGRAPH Computer Graphics 12, 3: 210-216.

22. Ken Hinckley, Xiang 'anthony Chen, and Hrvoje Benko. 2013. Motion and context sensing techniques for pen computing. Proceedings of Graphics Interface 2013: 71-78.

23. David Holman, Roel Vertegaal, Mark Altosaar, Nikolaus Troje, and Derek Johns. 2005. Paper windows: interaction techniques for digital paper. Proceedings of SIGCHI conference on human computer interaction: 591-599.

24. Stéphane Huot, Mathieu Nancel, and Michel Beaudouin-Lafon. 2008. Pushmenu: Extending marking menus for pressure-enabled input devices.

25. Lynette A Jones and Susan J Lederman. 2006. Human Hand Function. Oxford University Press, USA.

26. Johan Kildal and Marion Boberg. 2013. Feel the Action: Dynamic Tactile Cues in the Interaction with Deformable UIs. CHI '13 Extended Abstracts on Human Factors in Computing Systems: 1563-1568.

27. K S C Kuang, W J Cantwell, and P J Scully. 2002. An evaluation of a novel plastic optical fibre sensor for axial strain and bend measurements. Measurement Science and Technology 13, 10: 1523.

28. Gordon Kurtenbach and William Buxton. 1994. User learning and performance with marking menus. Proceedings of the SIGCHI conference on Human Factors in computing systems: 258-264.

29. Byron Lahey, Audrey Girouard, Winslow Burleson, and Roel Vertegaal. 2011. PaperPhone: understanding the use of bend gestures in mobile devices with flexible electronic paper displays. Proceedings of the SIGCHI conference on Human Factors in computing systems: 1303-1312.

30. Sana Maqsood, Sonia Chiasson, and Audrey Girouard. 2016. Bend Passwords: using gestures to authenticate on flexible devices. Pers. Ubiquit. Comput. 20, 4: 573-600.

31. Microsoft. Surface Pro 2. Retrieved from https://www.microsoft.com/surface/en$\mathrm{ca} /$ devices/surface-pro-2

32. Jon Peddie. 2013. The history of visual magic in computers: how beautiful images are made in $C A D, 3 D, V R$ and $A R$. Springer.

33. Gonzalo A Ramos and Ravin Balakrishnan. 2007. Pressure Marks. Proceedings of the SIGCHI conference on Human Factors in computing systems: 1375-1384. 
34. Gonzalo Ramos and Ravin Balakrishnan. 2005. Zliding: fluid zooming and sliding for high precision parameter manipulation. Proceedings of the 18th annual ACM symposium on User interface software and technology: 143-152.

35. Gonzalo Ramos, Matthew Boulos, and Ravin Balakrishnan. 2004. Pressure Widgets. Proceedings of the SIGCHI conference on Human Factors in computing systems: 487-494.

36. Anne Roudaut, Abhijit Karnik, Markus Löchtefeld, and Sriram Subramanian. 2013. Morphees: toward high "shape resolution" in self-actuated flexible mobile devices. Proceedings of the SIGCHI conference on Human Factors in computing systems: 593.

37. Eric Saund and Edward Lank. 2003. Stylus input and editing without prior selection of mode. Proceedings of the 16th annual ACM symposium on User interface software and technology: 213-216.

38. Eric Singer. 2003. Sonic banana: a novel bend-sensor-based MIDI controller. Proceedings of the 2003 conference on New interfaces for musical expression: 220-221.

39. Ronit Slyper, Ivan Poupyrev, and Jessica Hodgins. 2011. Sensing Through Structure: Designing Soft Silicone Sensors. Proceedings of the Fifth International Conference on Tangible, Embedded, and Embodied Interaction: 213-220.

40. Hyunyoung Song, Hrvoje Benko, Francois Guimbretiere, Shahram Izadi, Xiang Cao, and Ken Hinckley. 2011. Grips and gestures on a multi-touch pen. Proceedings of the SIGCHI conference on Human Factors in computing systems: 1323-1332.

41. Steve Strassmann. 1986. Hairy brushes. Proceedings of the 13th annual conference on Computer graphics and interactive techniques: 225-232.

42. Paul Strohmeier, Jesse Burstyn, Juan Pablo Carrascal, Vincent Levesque, and Roel Vertegaal. 2016. ReFlex: A Flexible Smartphone with Active Haptic Feedback for Bend Input. Proceedings of the TEI '16: Tenth International Conference on Tangible, Embedded, and Embodied Interaction: 185-192.

43. Minghui Sun, Xiang Cao, Hyunyoung Song, et al. 2011. Enhancing naturalness of pen-and-tablet drawing through context sensing. Proceedings of the ACM International Conference on Interactive Tabletops and Surfaces: 83-86.

44. Ivan E Sutherland. 1964. Sketch pad a man-machine graphical communication system. Proceedings of the SHARE design automation workshop: 6.329-6.346.

45. Yu Suzuki, Kazuo Misue, and Jiro Tanaka. 2010. Interaction Technique Combining Gripping and Pen Pressures. International Conference on KnowledgeBased and Intelligent Information \& Engineering Systems: 440-448.

46. Aneesh P Tarun, Byron Lahey, Audrey Girouard, Winslow Burleson, and Roel Vertegaal. 2011. Snaplet: Using Body Shape to Inform Function in Mobile Flexible Display Devices. CHI '11 Extended Abstracts on Human Factors in Computing Systems: 329-334.

47. Feng Tian, Lishuang Xu, Hongan Wang, et al. 2008. Tilt Menu: Using the 3D Orientation Information of Pen Devices to Extend the Selection Capability of Penbased User Interfaces. Proceedings of the SIGCHI conference on Human Factors in computing systems:1371-1380.

48. Giovanni Maria Troiano, Esben Warming Pedersen, and Kasper Hornbæk. 2015. 
Deformable Interfaces for Performing Music. Proceedings of the 33rd Annual ACM Conference on Human Factors in Computing Systems: 377-386.

49. R Vertegaal and I Poupyrev. 2008. Organic user interfaces. Commun. ACM 51, 6: 48-55.

50. Daniel Vogel and Géry Casiez. 2011. Conté: multimodal input inspired by an artist's crayon. Proceedings of the 24th annual ACM symposium on User interface software and technology: 357-366.

51. Wacom. 2016. Airbrush Pen. Retrieved from https://usstore.wacom.com/Product/Airbrush-Pen-black-S01\#/undefined1

52. Kristen Warren, Jessica Lo, Vaibhav Vadgama, and Audrey Girouard. 2013. Bending the rules: bend gesture classification for flexible displays. Proceedings of the SIGCHI conference on Human Factors in computing systems: 607-610.

53. Jacob O Wobbrock, Edward Cutrell, Susumu Harada, and I Scott MacKenzie. 2008. An Error Model for Pointing Based on Fitts' Law. Proceedings of the SIGCHI conference on Human Factors in computing systems: 1613-1622.

54. Jacob O Wobbrock, Andrew Wilson, and Yang Li. \$1 Unistroke Recognizer.

55. Z Xiaolei. 2014. Comparative Study on Cursor Position Controlled by Pen Pressure and Pen Tilt. International Symposium on Computational Intelligence and Design: 375-378.

56. Yizhong Xin, Xiaojun Bi, and Xiangshi Ren. 2011. Acquiring and Pointing: An Empirical Study of Pen-tilt-based Interaction. Proceedings of SIGCHI conference on Human Factors in computing systems: 849-858. 